\title{
THE SCHUR AND (WEAK) DUNFORD-PETTIS PROPERTIES IN BANACH LATTICES
}

\author{
ANNA KAMIŃSKA and MIECZYSLAW MASTYLO
}

(Received 13 November 2000)

Communicated by A. Pryde

\begin{abstract}
We study the Schur and (weak) Dunford-Pettis properties in Banach lattices. We show that $\ell_{1}, c_{0}$ and $\ell_{\infty}$ are the only Banach symmetric sequence spaces with the weak Dunford-Pettis property. We also characterize a large class of Banach lattices without the (weak) Dunford-Pettis property. In MusielakOrlicz sequence spaces we give some necessary and sufficient conditions for the Schur property, extending the Yamamuro result. We also present a number of results on the Schur property in weighted Orlicz sequence spaces, and, in particular, we find a complete characterization of this property for weights belonging to class $\Lambda$. We also present examples of weighted Orlicz spaces with the Schur property which are not $\mathscr{L}_{1}$-spaces. Finally, as an application of the results in sequence spaces, we provide a description of the weak Dunford-Pettis and the positive Schur properties in Orlicz spaces over an infinite non-atomic measure space.
\end{abstract}

2000 Mathematics subject classification: primary 46B20, 46E30, 46B42, 46B45.

\section{Introduction}

A Banach space $X$ is said to have the Dunford-Pettis property, shortly (DP)-property or $X \in(\mathrm{DP})$, if for all weakly null sequences $\left(x_{n}\right)$ in $X$ and $\left(f_{n}\right)$ in $X^{*}$ (topological dual), we have $f_{n}\left(x_{n}\right) \rightarrow 0$, or equivalently, if every weakly compact operator from $X$ into an arbitrary Banach space $Y$ is a Dunford-Pettis operator. Recall that an operator $T: X \rightarrow Y$ between two Banach spaces is a Dunford-Pettis operator, whenever $T$ maps weakly null sequences into norm null sequences. A continuous operator $T$ mapping a Banach lattice $E$ into a Banach space $Y$ is called almost Dunford-Pettis if $\left\|T x_{n}\right\| \rightarrow 0$ for every weakly null sequence $\left(x_{n}\right)$ consisting of pairwise disjoint

The first author was supported by NATO Grant CRG 972918.

The second author was supported by NATO Grant CRG 972918 and KBN Grant 2 P03A 04218.

(C) 2002 Australian Mathematical Society 1446-7887/2000 $\$ A 2.00+0.00$ 
elements. We will say that a Banach lattice $E$ has the weak Dunford-Pettis property (shortly (wDP)-property or $E \in$ (wDP)) (respectively, positive Schur property) if every weakly compact operator on $E$ is almost Dunford-Pettis (respectively, every weakly null sequence with positive terms is norm null). It is clear that every Banach space $X$ with the Schur property ( $X \in(\mathrm{SP})$ ), that is, weak null sequences in $X$ are norm null, (respectively, positive Schur property), has the (DP)-property (respectively, the (wDP)-property). For equivalent definitions and various characterizations of the (weak) Dunford-Pettis and the (positive) Schur properties we refer to $[2,6,33,34]$ and $[30,34]$, respectively.

In [15], a complete description of symmetric spaces with the Dunford-Pettis property on $\mathbb{N}$ as well as on $(0, a), 0<a \leq \infty$ has been given (see also [28] for symmetric function spaces on $[0,1])$. In the case of symmetric spaces on $(0, a)$ there are only six (respectively, two) symmetric spaces with the (DP)-property whenever $a=\infty$ (respectively, $a<\infty$ ). If $a<\infty$ then the only symmetric spaces with the (DP)-property are $L_{1}$ and $L_{\infty}$. It is known that there are more symmetric spaces with (wDP)-property, for example Orlicz and Lorentz spaces on the interval $(0,1)$ (see $[20,33,34]$ ).

In the case of Banach function spaces without symmetry the situation seems to be more complicated, however a few results are already known for example a characterization of the Schur and Dunford-Pettis properties in Nakano sequence spaces (see $[8,32]$ ). It appears that almost without modifications in proofs (see [32]), the Nakano space $\ell^{\left(p_{n}\right)}$ has (wDP)-property if and only if it has (DP)-property, that is, the only accumulation points of $\left(p_{n}\right)$ are 1 or $\infty$. Further objects of investigation in that direction are Musielak-Orlicz spaces. Only a little has been known about the Schur property for these spaces (see Yamamuro results [36]). In this paper, among others, we give an extension of the Yamamuro results finding more accurate conditions which assure the Schur property for some class of Musielak-Orlicz sequence spaces.

The article is divided into 6 sections. After introductory Section 1, in Section 2, we collect definitions, notations and some auxiliary results used in the sequel.

In Section 3 we study (DP)- and (wDP)-properties in Banach lattices. Applying the well known construction of Davis-Figiel-Johnson-Pełczyński [5] we characterize a large class of Banach lattices without the Dunford-Pettis property and in the case of sequence spaces also without the weak Dunford-Pettis property. Employing then this result we show that (DP)- and (wDP)- properties coincide in the class of symmetric sequence spaces. We finish this section with a corollary on the Schur property in the so called symmetrization of Banach spaces with a basis.

Section 4 consists of a number of results on the Schur property of the sequence Musielak-Orlicz spaces. Some sufficient conditions are presented in Theorem 4.4 with its corollary Theorem 4.8, as well as in Theorem 4.7, representing two different approaches to the problem. We also state some necessary conditions and we recover the results obtained by Halperin and Nakano in [8], and Yamamuro in [36]. We also 
show, by providing examples, that our results are sharper. We were not able, however, to find a complete description of the Schur property in these spaces, which should not be surprising in view of the attempts we pursue in Section 5, where we study weighted Orlicz sequence spaces, a particular class of Musielak-Orlicz spaces. Even for this comparatively narrow class of Musielak-Orlicz spaces the description of (SP) is a quite complicated task, which can be seen in Theorem 5.3, where we characterize completely the Schur property for the weights belonging to the class $\Lambda$, in terms of the Orlicz functions from the class $C_{\phi}^{\infty}$ (see $\left.[9,23,27]\right)$. For weights belonging to classes $\Lambda_{1}$ and $\Lambda_{\infty}$, we obtain some partial results too.

Finally, in short Section 6, applying the results from Section 5, we characterize the positive (SP) and the (wDP)-property in Orlicz spaces $L_{\phi}(\mu)$ over infinite and non-atomic measure spaces.

\section{Definitions and notations}

We follow throughout standard Banach space terminology. For unexplained notation the reader is referred to [2] and [23]. However, we want to explain some frequently used terms and agree on some notations. Let further $E$ denote a Banach lattice and $E^{+}=\{x \in E: x \geq 0\}$ its positive cone. Recall that $E$ is said to have the Fatou property if whenever $\left(x_{n}\right)$ is a norm bounded sequence in $E$ such that $0 \leq x_{n} \uparrow x=\sup x_{n}$, then $x \in E$ and $\lim _{n}\left\|x_{n}\right\|=\|x\|$. An element $x \in E$ is said to have an order continuous norm if for every sequence $x_{n} \downarrow 0$ in $E$ with $x_{n} \leq x$, we have $\left\|x_{n}\right\| \rightarrow 0$. The norm in a Banach lattice $E$ is called order continuous if every element in $E$ has order continuous norm and the largest ideal consisting of all elements with order continuous norms will be denoted by $E_{a}$.

Let $(\Omega, \mathscr{B}, \mu)$ (or shortly $(\Omega, \mu)$ ) be a $\sigma$-finite measure space. Throughout the paper $\mu$ will be always either non-atomic or purely atomic, that is, $\Omega=\mathbb{N}$ and $\mu(\{n\})=1$ for each $n \in \mathbb{N}$. By $L^{0}=L^{0}(\mu)$ denote a vector lattice of all (equivalence classes) of $\mu$-measurable real-valued functions defined on $\Omega$, equipped with the topology of convergence in measure on $\mu$-finite sets. A Banach space $E$ is said to be a Banach lattice on $(\Omega, \mu)$ if $E$ is a subspace in $L^{0}$ with the following two properties:

(i) $|x| \leq|y|, y \in E$ implies $x \in E$ and $\|x\| \leq\|y\|$;

(ii) there exists $u \in E$ such that $u>0$ on $\Omega$.

In what follows a Banach lattice on $\mathbb{N}$ will be called a Banach sequence space. The elements of a Banach sequence space will be further denoted as usual by $x=\left(x_{n}\right)$, but occasionally we will also use the notation $x=(x(n))$, where $x(n) \in \mathbb{R}$ and $n \in \mathbb{N}$. The Köthe dual $E^{\prime}$ of a Banach lattice $E$ is then defined as

$$
E^{\prime}=\left\{x \in L^{0}:\|x\|_{E^{\prime}}=\sup _{\|y\|_{E} \leq 1} \int_{\Omega}|x y| d \mu<\infty\right\},
$$


and $E^{\prime}$ is a Banach lattice under the norm $\|\cdot\|_{E^{\prime}}$. The space $E_{c}^{*}$ of order bounded and order continuous functionals on $E$ is lattice isometric to the Köthe dual $E^{\prime}$ ([16]), which will be further denoted by $E_{c}^{*} \simeq E^{\prime}$. In particular, if $E$ has order continuous norm then the dual space $E^{*}$ can be naturally identified with $E^{\prime}$.

A Banach lattice on $(\Omega, \mu)$ is said to be symmetric if whenever $x \in E, y \in L^{0}$, and $x$ and $y$ are equimeasurable, then $y \in E$ and $\|x\|_{E}=\|y\|_{E}$. Recall that $x$ and $y$ are equimeasurable if they have identical distributions, that is, $\mu_{x}(\lambda)=\mu(\{t \in \Omega$ : $|x(t)|>\lambda\})=\mu_{y}(\lambda)$ for all $\lambda \geq 0$. Given an $x \in L^{0}$, by $x^{*}$ we denote its nonincresing rearrangement, that is, $x^{*}(t)=\inf \left\{\lambda \geq 0: \mu_{x}(\lambda) \leq t\right\}, t \geq 0$, under the convention $\inf \emptyset=0$. Obviously $x^{*}$ is a Lebesgue measurable function defined on the interval $(0, \mu(\Omega))$, and $x$ and $x^{*}$ are equimeasurable in the sense that $\mu_{x}(\lambda)=m_{x^{*}}(\lambda)$ for all $\lambda \geq 0$, where $m$ is the Lebesgue measure on $(0, \infty)$.

Given a non-atomic measure space $(\Omega, \mu)$ with $\mu(\Omega)<\infty$, we define Rademacher functions $\left(r_{n}\right)$ on $\Omega$ as a sequence of independent random variables with $\mu(\{s \in \Omega$ : $\left.\left.r_{n}(s)=1\right\}\right)=\mu\left(\left\{s \in \Omega: r_{n}(s)=-1\right\}\right)=\mu(\Omega) / 2$ for all $n \in \mathbb{N}$.

In what follows we agree on some notations and provide auxiliary facts from interpolation theory (see $[4,19])$. A pair $\bar{X}=\left(X_{0}, X_{1}\right)$ of Banach spaces is called a Banach couple if $X_{0}$ and $X_{1}$ are both continuously embedded in a Hausdorff topological vector space $\mathscr{X}$. For a Banach couple $\bar{X}=\left(X_{0}, X_{1}\right)$, the algebraic sum $X_{0}+X_{1}$ and the intersection $X_{0} \cap X_{1}$ will be denoted by $\Sigma(\bar{X})$ and $\Delta(\bar{X})$, respectively. They are both Banach spaces with the norms $\|x\|_{\Sigma(\bar{X})}=K(1, x ; \bar{X})$ and $\|x\|_{\Delta(\bar{X})}=\max \left\{\|x\|_{x_{0}},\|x\|_{x_{1}}\right\}$, respectively, where

$$
K(t, x ; \bar{X})=\inf \left\{\left\|x_{0}\right\|_{x_{0}}+t\left\|x_{1}\right\|_{X_{1}}: x=x_{0}+x_{1}\right\}, \quad t>0 .
$$

If $\left(X_{0}, X_{1}\right)$ is a Banach couple and $0<\theta<1,1 \leq p<\infty$, then the real method Lions-Peetre space

$$
\left(X_{0}, X_{1}\right)_{\theta, p}=\left\{x \in X_{0}+X_{1}:\|x\|_{\theta, p}=\left(\sum_{n=-\infty}^{\infty}\left(K\left(2^{n}, x ; \bar{X}\right) / 2^{n \theta}\right)^{p}\right)^{1 / p}<\infty\right\}
$$

is an intermediate Banach space between $X_{0}$ and $X_{1}$.

\section{Dunford-Pettis and weak Dunford-Pettis properties in Banach lattices}

In this section we show that a quite large class of Banach lattices fail the DunfordPettis property. At first we shall consider Banach lattices defined on non-atomic measure spaces and next we will investigate Banach sequence spaces.

Further we shall employ the following well known construction of Davis-FigielJohnson-Pełczyński [5]. Let $X$ be a Banach space and $W$ be a convex, circled, norm 
bounded subset of $X$. For each $n \in \mathbb{N}$, set $U_{n}=W+2^{-n} B_{X}$, where $B_{X}$ is the unit ball of $X$, and denote by $p_{n}$ the Minkowski functional of $U_{n}$, that is,

$$
p_{n}(x)=\inf \left\{\lambda>0: x \in \lambda U_{n}\right\}
$$

For $0<\theta<1$ and $1<q<\infty$, let

$$
Y=Y_{\theta, q}:=\left\{x \in X:\|x\|=\left(\sum_{n=1}^{\infty}\left(2^{-n \theta} p_{n}(x)\right)^{q}\right)^{1 / q}<\infty\right\} .
$$

It was shown in [5] that $Y_{1 / 2,2}$ is reflexive if and only if $W$ is a relatively weakly compact subset of $X$. Note that if $X$ is a Banach lattice and $W$ is also a solid set, then the Banach space $Y_{\theta, p}$ is itself a Banach lattice (see [2, page 297]).

In what follows we will also need the well-known result of Levy [21] which says that if $\left(X_{0}, X_{1}\right)$ is a non-trivial Banach couple, that is, $\Delta(\bar{X})$ is a non-closed subspace of $\Sigma(\bar{X})$, then for any $0<\theta<1$ and $1 \leq q \leq \infty$ the Lions-Peetre interpolation space $\left(X_{0}, X_{1}\right)_{\theta, q}$ contains a complemented subspace isomorphic to $\ell_{q}$.

It is easy to see that if $X_{0} \hookrightarrow X_{1}$, then the Lions-Peetre space $\left(X_{0}, X_{1}\right)_{\theta, q}$ with $1<$ $q<\infty$ coincides with the Davis-Figiel-Johnson-Pełczyński space $Y_{\theta, q}$ constructed under $X=X_{1}$ and $W=B_{X_{0}}$.

In the sequel we will need the following technical result.

PROPOSITION 3.1. Let $X$ be a Banach lattice on non-atomic measure space $(\Omega, \mu)$. Assume that there exist $x \in X_{a}$ and $y \in\left(X^{\prime}\right)_{a}$ such that $\mu(\operatorname{supp} x \cap \operatorname{supp} y)>0$. Then $X$ does not have the Dunford-Pettis property.

ProOF. Let $A=\operatorname{supp} x \cap \operatorname{supp} y$ with $\mu(A)>0$ and let us consider a Banach lattice $E=\left.X\right|_{A}$ defined on an induced non-atomic measure space $\left(A,\left.\Sigma\right|_{A}, v\right)$ with $v=\left.\mu\right|_{A}$. We have $u=x \chi_{A} \in E_{a}, v=y \chi_{A} \in\left(E^{\prime}\right)_{a}$, and supp $u=\operatorname{supp} v=A$. Now, since $E_{a}$ has order continuous norm, order interval $W=[-u, u]$ is a weakly compact set in $E_{a}$ and thus in $E$. By the Davis et all construction in [5], there exists a reflexive Banach lattice $Y$ continuously imbedded into $E$. Similarly, we conclude that there exists a reflexive Banach lattice $Z$ such that $Z \hookrightarrow E^{\prime}$. Hence $E^{\prime \prime} \hookrightarrow Z^{\prime}$ with $Z^{\prime}$ being reflexive. Since $E \hookrightarrow E^{\prime \prime}$, we have $Y \hookrightarrow E \hookrightarrow Z^{\prime}$. For a Banach lattice $Y \cap Z$, we have $\operatorname{supp}(Y \cap Z)=A$. Thus, it follows by [16, Corollary 2, page 95], that there exists a sequence $\left(A_{n}\right)$ of pairwise disjoint sets in $\left.\Sigma\right|_{A}$ such that $\bigcup_{n=1}^{\infty} A_{n}=A$ and $\chi_{A_{n}} \in Y \cap Z$. Since $Y \cap Z \hookrightarrow E$ and $Y \cap Z \hookrightarrow Z^{\prime \prime}$, we clearly obtain that for some measurable set $B$ with $0<\mu(B)<\infty,\left.\left.\left.\left.\left.L_{\infty}\right|_{B} \hookrightarrow Y\right|_{B} \hookrightarrow E\right|_{B} \hookrightarrow Z^{\prime}\right|_{B} \hookrightarrow L_{1}\right|_{B}$. Now if we assume that $X \in(\mathrm{DP})$, then $\left.E\right|_{B} \in$ (DP) as a complemented subspace of $X$. Since $\left.Y\right|_{B}$ and $\left.Z^{\prime}\right|_{B}$ are reflexive spaces, we obtain that the inclusion map $\left.\left.L_{\infty}\right|_{B} \hookrightarrow L_{1}\right|_{B}$ is compact, which is a contradiction. Indeed, since $\mu$ is atomless 
we can construct on $B$ a sequence $\left(r_{n}\right)$ of Rademacher functions. Of course, $\left(r_{n}\right)$ is bounded in $\left.L_{\infty}\right|_{B}$ and $r_{n} \rightarrow 0$ weakly in $\left.L_{1}\right|_{B}$ but $\left\|r_{n}\right\|_{L_{1}} \nrightarrow 0$. This finishes the proof.

REMARK. Let us observe that the assumption $\mu(\operatorname{supp} x \cap \operatorname{supp} y)>0$ is essential in the above proposition. In fact, let

$$
X=\left\{x \in L^{0}((0,2), m):\|x\|=\left\|x \chi_{(0,1)}\right\|_{L_{1}(0,1)}+\left\|x \chi_{(1,2)}\right\|_{L_{\infty}(1,2)}<\infty\right\} .
$$

Then we have $X_{a}=L_{1}(0,1)$ and $\left(X^{\prime}\right)_{a}=L_{1}(1,2)$. Since $X$ is isomorphic to $L_{1}(0,1) \oplus_{1} L_{\infty}(1,2), X$ has the Dunford-Pettis property.

Note that in general, if $X$ is a Banach lattice such that $X_{a}=\left(X^{\prime}\right)_{a}=\{0\}$ then this does not imply that $X$ has the Dunford-Pettis property. To see this let us recall that if $E$ and $F$ are two Banach lattices defined on $\left(\Omega_{1}, \mu_{1}\right)$ and $\left(\Omega_{2}, \mu_{2}\right)$ respectively, then a space with mixed norm $E_{s}\left[F_{t}\right]$ (respectively, $F_{t}\left[E_{s}\right]$ ) is defined to be a Banach lattice of $x \in L^{0}\left(\Omega_{1} \otimes \Omega_{2}, \mu_{1} \otimes \mu_{2}\right)$ with the norm $\|x\|=\|\| x(s, t)\left\|_{F, s}\right\|_{E, t}$ (respectively, $\left.\|x\|=\|\| x(s, t)\left\|_{E, t}\right\|_{F, s}\right)($ see $[16$, page 310]).

PROPOSITION 3.2. For any $1<p<\infty$ there exists a Banach lattice $X=X(p)$ such that $X_{a}=\left(X^{\prime}\right)_{a}=\{0\}$ and $X$ (respectively, $\left.X^{\prime}\right)$ contains a complemented subspace isomorphic to $\ell_{p}$ (respectively, to $\ell_{q}$ ), with $1 / p+1 / q=1$.

PROOF. For any couple $(E, F)$ of Banach lattices we have (see [25, Theorem 3.1])

$$
\left((E, F)_{\theta, p}\right)^{\prime}=\left(E^{\prime}, F^{\prime}\right)_{\theta, q}
$$

for any $0<\theta<1$ and $1<p<\infty$, where $1 / p+1 / q=1$. It is easily seen that if $(E, F)$ is a nontrivial couple then also $\left(E^{\prime}, F^{\prime}\right)$ is a nontrivial couple. Thus, if $(E, F)$ is nontrivial, it follows from the Levy's result that $(E, F)_{\theta, p}$ (respectively, $\left.\left((E, F)_{\theta, p}\right)^{\prime}\right)$ contains a complemented subspace isomorphic to $\ell_{p}$ (respectively, to $\left.\ell_{q}\right)$. In order to finish the proof it suffices to take $X=(E, F)_{\theta, p}$, where $(E, F)$ is a nontrivial couple of Banach lattices such that $E \hookrightarrow F$ and $\left(E^{\prime}\right)_{a}=F_{a}=\{0\}$. In fact by $E \hookrightarrow(E, F)_{\theta, p}=X \hookrightarrow F$, it follows that $X^{\prime} \hookrightarrow E^{\prime}$. Thus we obtain that $X_{a}=\left(X^{\prime}\right)_{a}=\{0\}$.

Let $\left(\Omega_{j}, \mu_{j}\right),(j=1,2)$ be non-atomic probability measure spaces. Take $E=$ $L_{s}^{1}\left[L_{t}^{\infty}\right]$ and $F=L_{t}^{\infty}\left[L_{s}^{1}\right]$. Then by [31], we have that $E \hookrightarrow F$ and $\left(E^{\prime}\right)_{a}=F_{a}=\{0\}$. It is easily seen that $(E, F)$ forms a nontrivial couple of Banach lattices. This completes the proof.

Since for nontrivial Banach couples $\left(X_{0}, X_{1}\right),\left\{\left(X_{0}, X_{1}\right)_{\theta, p}: 0<\theta<1,1 \leq p \leq \infty\right\}$ forms a family of different spaces for different parameters $\theta, p$ (see [12]), we obtain the following. 
COROLlaRY 3.3. There exists uncountable family $\left\{X_{\theta}: \theta \in(0,1)\right\}$ of Banach lattices on non-atomic probability measure space such that $\left(X_{\theta}\right)_{a}=\left(X_{\theta}\right)_{a}^{\prime}=\{0\}$ and $X_{\theta},\left(X_{\theta}\right)^{\prime}$ contain complemented copies of Hilbert space $\ell_{2}$, so $X_{\theta} \notin$ (DP) and $\left(X_{\theta}\right)^{\prime} \notin(\mathrm{DP})$, for any $0<\theta<1$.

PROPOSITION 3.4. Let $E$ be a Banach sequence space such that $\ell_{1} \subset E, E^{\prime} \subset c_{0}$ and $E^{\prime \prime} \subset c_{0}$. Then $E$ does not have the weak Dunford-Pettis property and in consequence it fails the Dunford-Pettis property.

PROOF. Assume for a contrary that $E \in$ (wDP). By the closed graph theorem, it follows that the imposed inclusions are continuous. Since $\ell_{1} \hookrightarrow E_{a}$ and the dual space $\left(E_{a}\right)^{*}$ can be identified with $\left(E_{a}\right)^{\prime}=E^{\prime}$, we conclude that $e_{n} \rightarrow 0$ weakly in $E$, by $E^{\prime} \subset c_{0}$. Since $E \subset E^{\prime \prime} \subset c_{0}$, so $E \subset c_{0}$ and $e_{n} \rightarrow 0$ weakly in $E^{\prime}$. By the Krein-Šmulian theorem, we have that the convex circled hull of $\left(e_{n}\right)_{n=1}^{\infty}$ is a relatively weakly compact subset in $E^{\prime}$. Thus, by the Davis et all construction [5] there exists a reflexive Banach sequence space $Y$ such that $\ell_{1} \hookrightarrow Y \hookrightarrow E^{\prime}$. Hence

$$
E \hookrightarrow E^{\prime \prime} \hookrightarrow Y^{\prime} \hookrightarrow \ell_{\infty}
$$

which implies that the inclusion map $E \hookrightarrow \ell_{\infty}$ is weakly compact. Now by the (wDP)-property of $E$ and the fact that $e_{n} \rightarrow 0$ in $E,\left\|e_{n}\right\|_{\ell_{\infty}} \rightarrow 0$, which is a contradiction.

THEOREM 3.5. A symmetric sequence space $E$ has the weak Dunford-Pettis property if and only if it has the Dunford-Pettis property, that is, E coincides up to equivalence of norms with either $\ell_{1}$ or $c_{0}$ or $\ell_{\infty}$.

PROOF. Suppose that $\lim _{n \rightarrow \infty} \varphi_{E^{\prime}}(n)=C<\infty$, where $\varphi_{X}(n)=\left\|\chi_{(1, \ldots, n)}\right\|$ is a fundamental function of a symmetric space $X$. Then, we have $\sup _{n \geq 1}\left\|x_{n}\right\|_{E^{\prime}} \leq C$, where $x_{n}=\sum_{j=1}^{n} e_{j}$. Since $E^{\prime}$ has the Fatou property and $0 \leq x_{n} \uparrow \chi_{N}$, we get $\chi_{\mathrm{N}} \in E^{\prime}$. This yields $E^{\prime}=\ell_{\infty}$ and thus $E=\ell_{1}$.

If $\varphi_{E^{\prime}}(n) \rightarrow \infty$, as $n \rightarrow \infty$, then by an obvious inequality true for any symmetric sequence space $X$

$$
\varphi_{X}(n) x^{*}(n) \leq\|x\|_{X}, \quad x=(x(n)) \in X
$$

we have $E^{\prime} \hookrightarrow c_{0}$. Since $E \in(\mathrm{wDP})$, we conclude by Proposition 3.4 that $E^{\prime \prime}$ is not contained in $c_{0}$. This, in particular implies that $\lim _{n \rightarrow \infty} \varphi_{E^{\prime \prime}}(n)<\infty$. Now similarly as in part one, we get $E^{\prime \prime}=\ell_{\infty}$, which implies that $E^{\prime}=\ell_{1}$ and so $E=c_{0}$ or $E=\ell_{\infty}$. Since $\ell_{1}, c_{0}$ and $\ell_{\infty}$ have the Dunford-Pettis property the proof is finished. 
Now we present some consequences of the above results. Given a Banach space $(X,\|\cdot\|)$ with basis $\left(x_{n}\right)$, we define a norm $\|\cdot\| \|$ on the finitely supported elements $x=\sum_{n} a_{n} x_{n}$ of $X$ by

$$
\|x\|=\sup _{\pi \in \Pi}\left\|\sum_{n}\left|a_{n}\right| x_{\pi(n)}\right\|,
$$

where $\Pi$ is the family of all permutations of $\mathbb{N}$. Then $S(X)$, the so called symmetrization of $X$ with respect to $\left(x_{n}\right)$, is the $\|\cdot\| \mid \|$ completion of the space $\{x \in X$ : $x$ is finitely supported\}. Note that the vectors $\left(x_{n}\right)$ form a symmetric basis for $S(X)$ with symmetric basis constant 1 .

COROLLARY 3.6. Let $E$ be a Banach sequence space with semi-normalized basis $\left(e_{n}\right)$ of the unit standard basis vectors. If $E$ has the Schur property, then its symmetrization $S(E)$ with respect to $\left(e_{n}\right)$ coincides up to equivalence of norms with $\ell_{1}$.

ProOF. By the assumption that $\left(e_{n}\right)$ is semi-normalized, $\ell_{1} \hookrightarrow E \hookrightarrow c_{0}$, and hence $\ell_{1} \hookrightarrow S(E) \hookrightarrow E$. The Schur property of $E$ implies that $E^{\prime \prime}=E$ (otherwise $E$ would contain an isomorphic copy of $c_{0}$ ), and thus $E^{\prime \prime} \hookrightarrow c_{0}$. If $S(E)$ were not equal to $\ell_{1}$, then $S(E)^{\prime} \neq \ell_{\infty}$, and thus $S(E)^{\prime} \hookrightarrow c_{0}$. In consequence, we would get $E^{\prime} \hookrightarrow S(E)^{\prime} \hookrightarrow c_{0}$ and $E^{\prime \prime} \hookrightarrow c_{0}$. Since $E \in(\mathrm{SP})$, we obtain a contradiction by Proposition 3.4.

REMARK. Corollary 5.7 in Section 5 shows that in the above corollary the assumption that $\left(e_{n}\right)$ is a semi-normalized basis is essential.

\section{Schur property in Musielak-Orlicz sequence spaces}

A function $\phi: \mathbb{R}_{+} \rightarrow[0,+\infty]$ is said to be an Orlicz function if $\phi(0)=0, \phi$ is not identically equal to zero, and $\phi$ is convex and continuous at zero. By $\phi^{*}$ we denote its conjugate function in the sense of Young, that is,

$$
\phi^{*}(u)=\sup _{v>0}\{u v-\phi(v)\}, \quad u>0,
$$

and we notice that $\phi^{*}$ is again an Orlicz function. A sequence $\Phi=\left(\phi_{n}\right)$ of Orlicz functions $\phi_{n}$ is called a Musielak-Orlicz function and its Young's conjugate is a function $\Phi^{*}=\left(\phi_{n}^{*}\right)$. A Musielak-Orlicz space $\ell_{\Phi}$ is a collection of all sequences $x=\left(x_{n}\right)$ of real numbers such that for some $\lambda>0$

$$
I_{\Phi}(\lambda x)=\sum_{n=1}^{\infty} \phi_{n}\left(\lambda\left|x_{n}\right|\right)<\infty .
$$


It is well known that $\ell_{\Phi}$ equipped with the Luxemburg norm

$$
\|x\|_{\Phi}=\inf \left\{\lambda>0: I_{\Phi}(x / \lambda) \leq 1\right\}
$$

is a Banach sequence space with the Fatou property and its Köthe dual $\ell_{\Phi}^{\prime}$ coincides with $\ell_{\Phi^{*}}$. If for all $n \in \mathbb{N}, \phi_{n}=\phi$, where $\phi$ is an Orlicz function, then $\ell_{\phi}$ is a sequence Orlicz space. In particular, $\ell_{\phi}$ coincides with $\ell_{\infty}$ up to equivalence in norms whenever $\phi$ vanishes in a neighbourhood of zero.

It is said that a Musielak-Orlicz function $\Phi=\left(\phi_{n}\right)$ satisfies condition $\delta_{2}$ (in short $\left.\Phi \in\left(\delta_{2}\right)\right)$ if there exist $K, \beta>0$ and a sequence $\left(c_{n}\right) \in \ell_{1}^{+}$such that

$$
\phi_{n}(2 u) \leq K \phi_{n}(u)+c_{n}
$$

for every $u \in \mathbb{R}_{+}$and $n \in \mathbb{N}$ if $\phi_{n}(u) \leq \beta$.

Recall also that given Musielak-Orlicz functions $\Phi=\left(\phi_{n}\right)$ and $\Psi=\left(\psi_{n}\right)$, the spaces $\ell_{\Phi}$ and $\ell_{\Psi}$ coincide with equivalence of norms if and only if $\Phi$ is equivalent to $\Psi$ (we write $\Phi \sim \Psi$ ), that is, for some $K, \delta>0$ and $\left(c_{n}\right) \in \ell_{1}^{+}$it holds

$$
\phi_{n}(K u) \leq \psi_{n}(u)+c_{n} \text { and } \psi_{n}(K u) \leq \phi_{n}(u)+c_{n},
$$

for every $u \in \mathbb{R}_{+}$and $n \in \mathbb{N}$ such that the first inequality is satisfied if $\psi_{n}(u) \leq \delta$ and the second one holds if $\phi_{n}(u) \leq \delta$. More information on Musielak-Orlicz sequence spaces we can find in $[11,17,26,35]$ and $[23$, vol. I].

Following the idea of Matuszewska and Orlicz for Orlicz functions (see [24]) and the ideas contained in papers $[14,17]$ for Musielak-Orlicz functions, we define indices associated with sequence Musielak-Orlicz spaces.

Given $1 \leq p<\infty$ and $\Phi=\left(\phi_{n}\right)$, it is said that $\Phi$ satisfies condition $\delta^{p}$ (respectively, $\left.\delta^{* p}\right)$ [17] if for some $K, \delta>0$ and $\left(c_{n}\right) \in \ell_{1}^{+}$

$$
\phi_{n}(\lambda u) \leq K \lambda^{p}\left(\phi_{n}(u)+c_{n}\right), \quad\left(\text { respectively, } \phi_{n}(\lambda u) \geq K \lambda^{p}\left(\phi_{n}(u)-c_{n}\right),\right. \text { ) }
$$

for all $\lambda \geq 1, n \in \mathbb{N}$ and $u \in \mathbb{R}_{+}$if $\phi_{n}(\lambda u) \leq \delta$. Then the lower and upper indices $\alpha(\Phi)$ and $\beta(\Phi)$ are defined as follows

$$
\alpha(\Phi)=\sup \left\{p \geq 1: \Phi \in \delta^{* p}\right\}, \quad \beta(\Phi)=\inf \left\{p \geq 1: \Phi \in \delta^{p}\right\} .
$$

We observe that both conditions $\delta^{p}$ and $\delta^{* p}$ are preserved under equivalence of functions and moreover $\beta(\Phi)<\infty$ (respectively, $\alpha(\Phi)>1$ ) if and only if $\Phi \in\left(\delta_{2}\right)$ (respectively, $\Phi^{*} \in\left(\delta_{2}\right)$ ) [17].

Given an Orlicz function $\phi$ such that $\phi$ is finite, vanishes only at zero and its derivative $\phi^{\prime}$ exists, define

$$
a(\phi)=\inf _{0<u<\infty} \frac{u \phi^{\prime}(u)}{\phi(u)}, \quad b(\phi)=\sup _{0<u<\infty} \frac{u \phi^{\prime}(u)}{\phi(u)},
$$


known in the literature (see for example $[23,24]$ ) as lower and upper Simonenko indices respectively. The folowing equalities hold true

$$
\frac{1}{a(\phi)}+\frac{1}{b\left(\phi^{*}\right)}=1, \quad \frac{1}{b(\phi)}+\frac{1}{a\left(\phi^{*}\right)}=1 .
$$

In the sequel we will need a number of technical results. For the proof of (i) and (ii) of the next result see also [36].

THEOREM 4.1. Let $\Phi=\left(\phi_{n}\right)$ be an arbitrary Musielak-Orlicz function. Then the following hold true:

(i) $\ell_{1} \subset \ell_{\Phi}$ if and only if $\sup _{n} \phi_{n}(b)<\infty$ for some $b>0$;

(ii) $\ell_{\Phi} \subset \ell_{\infty}$ if and only if $\inf _{n} \phi_{n}(b)>0$ for some $b>0$;

(iii) $\ell_{\Phi} \subset c_{0}$ if and only if $\liminf _{n} \phi_{n}(b)>0$ for all $b>0$.

PROOF. (i) By the conditions on inclusions between Musielak-Orlicz spaces, $\ell_{1} \subset \ell_{\Phi}$ whenever for some $K>0$ and $\left(c_{n}\right) \in \ell_{1}^{+}, \phi_{n}(K u) \leq u+c_{n}$ for all $n \in \mathbb{N}, u \in \mathbb{R}_{+}$ such that $u \leq \delta$. Letting $b=K \delta$ it follows that $\sup _{n} \phi_{n}(b) \leq \delta+\sup _{n} c_{n}<\infty$. Now, if $\sup _{n} \phi_{n}(b)<\infty$ for some $b>0$ and $x=\left(x_{n}\right) \in \ell_{1}$ with $\|x\|_{\ell_{1}}=1$, then by convexity of $\phi_{n}, I_{\Phi}(b x) \leq \sup _{n} \phi_{n}(b)$, which implies that $x \in \ell_{\Phi}$.

(ii) If $\inf _{n} \phi_{n}(b)>0$ then $\phi_{n}(b) \geq B>0$ for all $n \in \mathbb{N}$ and some $B>0$. Given any $x=\left(x_{n}\right) \in \ell_{\Phi}$, for some $\lambda>0$ and sufficiently large $n, \phi_{n}\left(\lambda\left|x_{n}\right|\right) \leq B \leq \phi_{n}(B)$. Hence $x \in \ell_{\infty}$.

Now if we suppose that $\inf _{n} \phi_{n}(u)=0$ for all $u \in \mathbb{R}_{+}$, then for any sequence $0<x_{k} \rightarrow \infty$ there exists a subsequence $\left(n_{k}\right)$ such that $\sum_{k=1}^{\infty} \phi_{n_{k}}\left(x_{k}\right)<\infty$. Setting $x=\left(x_{n}\right)$ with $x_{n}=x_{k}$ for $n=n_{k}$ and $x_{n}=0$ otherwise, $x \notin \ell_{\infty}$ while $x \in \ell_{\Phi}$.

(iii) Assuming that lim inf $\phi_{n}(b)>0$ for all $b>0$ and letting $x$ be in $\ell_{\Phi}$, suppose that $x$ is not an element of $c_{0}$. Hence $\lim _{n} \phi_{n}\left(\lambda\left|x_{n}\right|\right)=0$ for some $\lambda>0$.

On the other hand, $\left|x_{n_{k}}\right|>\epsilon>0$ for a subsequence $\left(n_{k}\right)$, which implies that

$$
\liminf _{k} \phi_{n_{k}}\left(\lambda\left|x_{n_{k}}\right|\right) \geq \liminf _{k} \phi_{n_{k}}(\lambda \epsilon) \geq \liminf _{n} \phi_{n}(\lambda \epsilon)>0,
$$

which is a contradiction. Let now $\ell_{\Phi} \subset c_{0}$ and yet for some $b>0, \liminf _{n} \phi_{n}(b)=0$. Then for some subsequence $\left(n_{k}\right), \sum_{k=1}^{\infty} \phi_{n_{k}}(b)<\infty$. Hence $x=\left(x_{n}\right)$ with $x_{n_{k}}=b$ and zero otherwise, belongs to $\ell_{\Phi}$ but it is not an element of $c_{0}$.

In many cases it is convenient to deal with Musielak-Orlicz spaces generated by the functions $\Phi=\left(\phi_{n}\right)$ such that each $\phi_{n}$ and its conjugate $\phi_{n}^{*}$ are finite, and $\phi_{n}(1)=1$ for all $n \in \mathbb{N}$. The next result shows that up to isomorphism, it is a general situation.

Proposition 4.2. Given an arbitrary Musielak-Orlicz function $\Phi=\left(\phi_{n}\right)$, there exists a function $\Psi=\left(\psi_{n}\right)$ such that $\ell_{\Phi}$ and $\ell_{\Psi}$ are isomorphic and for all $n \in \mathbb{N}$, both $\psi_{n}$ and $\psi_{n}^{*}$ are finite valued functions and $\psi_{n}(1)=1$. 
PROOF. Let $a_{n}=\sup \left\{u \in \mathbb{R}_{+}: \phi_{n}(u) \leq 1\right\}$ and let $0<\lambda<1$. Define $\Gamma=\left(\gamma_{n}\right)$ as follows

$$
\gamma_{n}(u)= \begin{cases}\phi_{n}(u), & \text { if } 0 \leq u \leq \lambda a_{n} \\ b_{n} u+c_{n}, & \text { if } \lambda a_{n} \leq u \leq a_{n} \\ K_{n} u^{2}, & \text { if } u \geq a_{n}\end{cases}
$$

where $b_{n}=\left(1-\phi_{n}\left(\lambda a_{n}\right)\right) /\left(a_{n}-\lambda a_{n}\right), c_{n}=\left(\phi_{n}\left(\lambda a_{n}\right)-\lambda\right) /(1-\lambda)$ and $K_{n}=b_{n} / 2$. Obviously $\gamma_{n}\left(a_{n}\right)=1$ and $\gamma_{n}$ are finite. Since $\lim _{u \rightarrow \infty} \gamma_{n}(u) / u=\infty$, so $\gamma_{n}^{*}$ are also finite. Moreover, if $\|x\|_{\Phi}=1$ then $I_{\Phi}(x)=\sum_{n=1}^{\infty} \phi_{n}\left(\left|x_{n}\right|\right) \leq 1$ and hence $\lambda\left|x_{n}\right| \leq \lambda a_{n}$. So $I_{\Gamma}(\lambda x)=I_{\Phi}(\lambda x) \leq 1$, which implies that $\|x\|_{\Gamma} \leq \lambda^{-1}\|x\|_{\Phi}$. Analogously we can show that $\|x\|_{\Phi} \leq \lambda^{-1}\|x\|_{\Gamma}$. Hence $\ell_{\Phi}$ and $\ell_{\Gamma}$ coincide and $\Phi \sim \Gamma$. Finally, define $\Psi=\left(\psi_{n}\right)$ with $\psi_{n}(u)=\gamma\left(a_{n} u\right)$, and notice that $\ell_{\Psi}$ is isometric to $\ell_{\Gamma}$, and so isomorphic to $\ell_{\Phi}$.

LEMMA 4.3. Assume that $\phi_{n}(1)=1$ for every $n \in \mathbb{N}$ and that $\lim _{n} \phi_{n}^{*}(a)=0$ for some $a>0$. Then there exist $C>0$ and a nonnegative sequence $\left(a_{n}\right) \in c_{0}$ such that $C u \leq \phi_{n}(u) \leq u$, for all $n \in \mathbb{N}$ and $a_{n} \leq u \leq 1$. In consequence there exists $a$ subsequence $\left(\phi_{n_{k}}\right)$ of $\left(\phi_{n}\right)$ such that $\ell_{\left(\phi_{n_{k}}\right)}=\ell_{1}$.

PROOF. Let $p_{n}$ be a right-hand derivative of $\phi_{n}$ and $q_{n}$ be a right-hand derivative of $\phi_{n}^{*}$. It is well known that $p_{n}\left(q_{n}(u)\right) \geq u, u \geq 0$ (see [18]). In view of the inequality $(a / 2) q_{n}(a / 2) \leq \phi_{n}^{*}(a), \lim _{n} q_{n}(a / 2)=0$. Denoting $b_{n}=q_{n}(a / 2)$, $p_{n}\left(b_{n}\right)=p_{n}\left(q_{n}(a / 2)\right) \geq a / 2$. Then for all $u \geq 2 b_{n}$,

$$
\phi_{n}(u) \geq \int_{b_{n}}^{u} p_{n}(s) d s \geq p_{n}\left(b_{n}\right)\left(u-b_{n}\right) \geq a u / 4 .
$$

Setting $a_{n}=2 b_{n}$ and $C=a / 4$, we are done by $\phi_{n}(u) \leq u$ for every $0 \leq u \leq 1$ and $n \in \mathbb{N}$.

To complete the proof take any subsequence $\left(n_{k}\right) \subset \mathbb{N}$ such that $\left(a_{k}\right) \in \ell_{1}^{+}$. Then, we obtain $C u \leq \phi_{n_{k}}(u) \leq u$ for all $k \in \mathbb{N}$ and $a_{k} \leq u \leq 1$. It follows that $\left(\phi_{n_{k}}(u)\right) \sim u$, which yields $\ell_{\left(\phi_{n_{k}}\right)}=\ell_{1}$.

Now we are in a position to give a characterization of the Schur property for some class of Musielak-Orlicz sequence spaces $\ell_{\Phi}$. Before we state this result we introduce the following notation.

Given a Musielak-Orlicz function $\Phi=\left(\phi_{n}\right)$, a sequence of convex functions defined on $[0,1]$ by $\left(\sum_{j=n_{i}+1}^{n_{i+1}} \phi_{j}\left(t \alpha_{j}\right)\right)_{i=1}^{\infty}$ is called a $\Phi$-convex block (respectively, Ф-convex $c_{0}$-block) of $\left(\phi_{n}\right)$ if $\left(n_{i}\right)_{i=1}^{\infty}$ is an increasing subsequence of $\mathbb{N}_{0}=\mathbb{N} \cup\{0\}$ with $n_{1}=0$ and $\left(\alpha_{j}\right)$ is a sequence of positive numbers (respectively, $\left(\alpha_{n}\right) \in c_{0}^{+}$), such that $\sum_{j=n_{i}+1}^{n_{i+1}} \phi_{j}\left(\alpha_{j}\right)=1$ for each $i \in \mathbb{N}$. 
In what follows a Musielak-Orlicz function $\Phi=\left(\phi_{n}\right)$ is called normalized whenever $\phi_{n}(1)=1$ for every $n \in \mathbb{N}$.

THEOREM 4.4. Let $\Phi=\left(\phi_{n}\right)$ be an arbitrary Musielak-Orlicz function. Consider the following conditions.

(i) $\Phi$ is normalized with $\Phi \in\left(\delta_{2}\right), \lim _{n} \phi_{n}^{*}(a)=0$ for some $a>0$ and each $\Phi$-convex $c_{0}$-block of $\left(\phi_{n}\right)$ contains a subsequence equivalent to a linear function.

(ii) $\Phi \in\left(\delta_{2}\right)$ and any $\Phi$-convex block of $\left(\phi_{n}\right)$ contains a subsequence equivalent to a linear function.

(iii) $\ell_{\Phi}$ has the Schur property.

Then (i) implies (ii), and (ii) is equivalent to (iii).

PROOF. (i) implies (ii). Let $\left(\psi_{i}(t)\right)=\left(\sum_{j=n_{i}+1}^{n_{i+1}} \phi_{j}\left(t \alpha_{j}\right)\right)$ be an arbitrary $\Phi$-convex block of $\left(\phi_{n}\right)$. If $\left(\alpha_{j}\right) \in c_{0}$, the proof is finished. Otherwise, $\alpha_{j_{i}} \geq \epsilon$ for some increasing sequence $\left(j_{i}\right)$ in $\mathbb{N}$ and $\epsilon>0$. By passing to a subsequence of $\left(\psi_{i}\right)$, we may assume, by Lemma 4.3, that for each $i \in \mathbb{N}$ there exists $j_{i}$ such that $j_{i} \in$ $\left\{n_{i}+1, \ldots, n_{i+1}\right\}$ and the sequence of basis vectors $\left(e_{j_{i}}\right)_{i=1}^{\infty}$ in $\ell_{\Phi}$ is equivalent to the unit vector basis $\left(e_{i}\right)$ of $\ell_{1}$. Define a block basic sequence $\left(x_{i}\right)$ of $\left(e_{n}\right)$ by $x_{i}=\sum_{j=n_{i}+1}^{n_{i+1}} \alpha_{j} e_{j}, i \in \mathbb{N}$. Then there exists $A>0$ such that

$$
\sum_{i=1}^{n}\left|\xi_{i}\right| \geq\left\|\sum_{i=1}^{n} \xi_{i} x_{i}\right\|_{\Phi}=\left\|\sum_{i=1}^{n} \sum_{j=n_{i}+1}^{n_{i+1}} \xi_{i} \alpha_{j} e_{j}\right\| \geq \epsilon\left\|\sum_{i=1}^{n} \xi_{i} e_{j_{i}}\right\|_{\Phi} \geq \epsilon A \sum_{i=1}^{n}\left|\xi_{i}\right|
$$

for any finite sequence $\left(\xi_{i}\right)$ in $\mathbb{R}^{n}, n \in \mathbb{N}$. Thus we conclude that $\left(x_{i}\right)$ is equivalent to the unit vector basis $\left(e_{i}\right)$ of $\ell_{1}$. Since $\left(x_{i}\right)$ in $\ell_{\Phi}$ is equivalent to the unit vector basis $\left(e_{i}\right)$ in $\ell_{\Psi}$ with $\Psi=\left(\psi_{i}\right)$, we conclude that $\left(\psi_{i}\right)$ is equivalent to a linear function.

(ii) implies (iii). Assume that $\ell_{\Phi}$ fails the Schur property. Thus there exists a normalized weakly null sequence $\left(x_{i}\right)$ in $\ell_{\Phi}$. By the Bessaga and Pełczyński principle, $\left(x_{i}\right)$ contains a subsequence which is equivalent to a block basic sequence of $\left(e_{n}\right)$. Thus without loss of generality, we may assume that $\left(x_{i}\right)$ is a normalized weakly null block basic sequence of $\left(e_{n}\right)$ in $\ell_{\Phi}$, that is, $\left\|x_{i}\right\|_{\Phi}=1$ and $x_{i}=\sum_{j=n_{i}+1}^{n_{i+1}} \alpha_{j} e_{j}, i \in \mathbb{N}$, $0=n_{1}<n_{2}<\cdots, \alpha_{j} \in \mathbb{R}$. Since $\Phi \in\left(\delta_{2}\right), \sum_{j=n_{i}+1}^{n_{i+1}} \phi_{j}\left(\left|\alpha_{j}\right|\right)=1$. Thus $\left(\psi_{i}\right)$ with $\psi_{i}(t)=\sum_{j=n_{i}+1}^{n_{i+1}} \phi_{j}\left(t\left|\alpha_{j}\right|\right), t \geq 0$, is a $\Phi$-convex block of $\left(\phi_{i}\right)$. Hence by (ii), we get $\ell_{\left(\psi_{i_{k}}\right)}=\ell_{1}$ for some subsequence $\left(\psi_{i_{k}}\right)$ of $\left(\psi_{i}\right)$. Since a block basic sequence $\left(x_{i_{k}}\right)$ in $\ell_{\Phi}$ is equivalent to a unit vector basis $\left(e_{k}\right)$ in $\left.\ell_{\left(\psi_{i_{k}}\right.}\right)$, we obtain a contradiction with $x_{i} \rightarrow 0$ weakly.

(iii) implies (ii). Let $\left(\psi_{i}(t)\right)=\left(\sum_{j=n_{i}+1}^{n_{i+1}} \phi_{j}\left(\alpha_{j} t\right)\right)$ be any $\Phi$-convex block of $\left(\phi_{n}\right)$. Since $\ell_{\Phi} \in(\mathrm{SP}), \Phi \in\left(\delta_{2}\right)$. Hence $\left(u_{i}\right)$ with $u_{i}=\sum_{j=n_{i}+1}^{n_{i+1}} \alpha_{j} e_{j}, i \in \mathbb{N}$, is a normalized block basic sequence in $\ell_{\Phi}$. Since $\left\|u_{n}-u_{m}\right\|_{\Phi} \geq 1$ for all $n \neq m,\left(u_{n}\right)$ does not contain a weak Cauchy subsequence, by the Schur property of $\ell_{\Phi}$. This 
yields by the Rosenthal $\ell_{1}$-theorem that $\left(u_{i}\right)$ contains a subsequence $\left(u_{i_{k}}\right)$ which is equivalent to the unit vector basis of $\ell_{1}$. It is easy to see that $\left(u_{i_{k}}\right)$ is equivalent to the unit vector basis $\left(e_{k}\right)$ of $\ell_{\left(\psi_{i_{k}}\right)}$. Hence $\ell_{\left(\psi_{i_{k}}\right)}=\ell_{1}$, and in consequence $\left(\psi_{i_{k}}\right)_{k=1}^{\infty}$ is equivalent to a linear function. The proof is complete.

Note that the proof of the implication from (i) to (ii) yields the following corollary.

COROLlaRY 4.5. Let $\Phi=\left(\phi_{n}\right)$ be an arbitrary normalized Musielak-Orlicz function such that $\Phi \in\left(\delta_{2}\right)$ and $\lim _{n} \phi_{n}^{*}(a)=0$ for some $a>0$. Then the following statements are true.

(i) The continuous inclusion map $\ell_{\Phi} \hookrightarrow c_{0}$ is almost Dunford-Pettis operator.

(ii) Any block basic sequence $\left(\sum_{j=n_{i}+1}^{n_{i+1}} \alpha_{j} e_{j}\right)_{i=0}^{\infty}$ of $\left(e_{j}\right)$ with $\left(\alpha_{j}\right) \notin c_{0}$ contains a subsequence equivalent to the unit vector basis of $\ell_{1}$.

The next theorem gives the sufficient conditions for $\ell_{\Phi}$ to have the Schur property. Although it is not a necessary condition (see Proposition 5.6 and Example before), it is sharper than that one given by Yamamuro in [36] (see Remark after Theorem 5.1). Before we state this result we need to introduce another growth condition for a Musielak-Orlicz function and prove a technical lemma.

We say that a Musielak-Orlicz function $\Phi=\left(\phi_{n}\right)$ satisfies condition (S) (shortly $\Phi \in(S))$ if there exist $\delta>0,0<\lambda<1$ and $\left(A_{j}\right) \in \ell_{1}^{+},\left(n_{j}\right) \subset \mathbb{N}$ such that

$$
\sum_{j=1}^{\infty} \sum_{i \geq n_{j}} \sup _{\phi_{i}(u) \leq \delta}\left\{\phi_{i}(\lambda u)-A_{j} \phi_{i}(u)\right\}<\infty .
$$

Notice that property (S) is preserved under equivalence of Musielak-Orlicz functions. Indeed, if $\Phi \in(S)$ then for some $\delta>0,0<\lambda<1,\left(A_{j}\right) \in \ell_{1}^{+},\left(n_{j}\right) \subset \mathbb{N}$ and $\left(c_{i j}\right) \subset \mathbb{R}_{+}$such that $\sum_{j=1}^{\infty} \sum_{i \geq n_{j}} c_{i j}<\infty$ it holds

$$
\phi_{i}(\lambda u) \leq A_{j} \phi_{i}(u)+c_{i j}
$$

for all $i \geq n_{j}$ and $u \in \mathbb{R}_{+}$such that $\phi_{i}(u) \leq \delta$. By the conditions on equivalence of $\Phi$ and $\Psi$ it follows that $\psi_{i}(\lambda K u) \leq A_{j} \psi_{i}\left(K^{-1} u\right)+\left(A_{j}+1\right) c_{i}+c_{i j}$ if $i \geq n_{j}$, $\psi_{i}\left(K^{-1} u\right) \leq \delta$ and $\phi_{i}(u) \leq \delta$. Let now $k_{j}$ and $m$ be such that $\sum_{i \geq k_{j}} c_{i} \leq 1 / 2^{j}$ and $c_{i} \leq \delta / 2$ for $i \geq m$. Observe that if $i \geq m$ and $\psi_{i}(u) \leq \delta / 2$, then $\phi_{i}(K u) \leq$ $\delta / 2+c_{i} \leq \delta$. Thus setting $\beta=\max \left\{n_{j}, k_{j}, m\right\}$ and $d_{i j}=\left(A_{j}+1\right) c_{i}+c_{i j}$ we have

$$
\psi_{i}(\beta u) \leq A_{j} \psi_{i}(u)+d_{i j}
$$

for $i \geq m_{j}$ and $\left.\psi_{(} u\right) \leq \delta / 2$, where $\sum_{j=1}^{\infty} \sum_{i \geq m_{j}} d_{i j}<\infty$. This shows that $\Psi \in(\mathrm{S})$.

LEMMA 4.6. Let $\Phi=\left(\phi_{n}\right)$ be a normalized Musielak-Orlicz function. If for some $\delta>0$ and all $n \in \mathbb{N}, \phi_{n}^{*}\left(b_{n}\right)=\delta$, then $0<\inf _{n} b_{n} \leq \sup _{n} b_{n}<\infty$. 
PROOF. By Theorem 4.1, $\ell_{1} \subset \ell_{\Phi} \subset \ell_{\infty}$. Hence the Köthe dual $\ell_{\Phi}^{\prime}=\ell_{\Phi^{*}}$ has the similar property, that is $\ell_{1} \subset \ell_{\Phi^{*}} \subset \ell_{\infty}$. Thus applying Theorem 4.1 again, there exist $0<a \leq b<\infty$ such that $0<\inf _{n} \phi_{n}^{*}(a) \leq \sup _{n} \phi_{n}^{*}(b)<\infty$. Now, assume for a contrary that $\inf _{n} b_{n}=0$. Without loss of generality let $b_{n} \rightarrow 0$ as $n \rightarrow \infty$. By convexity of $\phi_{n}^{*}$ we have for sufficently large $n \in \mathbb{N}$,

$$
\frac{\phi_{n}^{*}(b)-\phi_{n}^{*}\left(b_{n}\right)}{b-b_{n}} \geq \frac{\phi_{n}^{*}\left(b_{n}\right)}{b_{n}},
$$

which yields that $\phi_{n}^{*}(b) \geq \delta b / b_{n}$, but it is a contradiction, since $\delta b / b_{n} \rightarrow \infty$.

Analogously assume that $\lim _{n} b_{n}=\infty$. Thus $\phi_{n}^{*}\left(b_{n}\right) / b_{n} \geq \phi_{n}^{*}(a) / a$ for sufficiently large $n$, and hence $0<\inf _{n} \phi_{n}^{*}(a) / a \leq \delta / b_{n} \rightarrow 0$ which completes the proof.

THEOREM 4.7. Let $\Phi=\left(\phi_{n}\right)$ be a normalized Musielak-Orlicz function such that all $\phi_{n}^{*}$ are finite valued functions. If $\Phi$ satisfies condition $\delta_{2}$ and $\Phi^{*}$ fulfils condition (S) then $\ell_{\Phi}$ has the Schur property.

PROOF. Assume for a contrary that condition (S) is fulfilled but there exists a sequence $x_{n}=\left(x_{n}(i)\right)_{i=1}^{\infty} \equiv\left(x_{n}(i)\right) \in \ell_{\Phi}$ which converges weakly but not strongly. We assume without loss of generality that $x_{n}$ are nonnegative with disjoint supports $S_{n}$ and $\left\|x_{n}\right\|_{\Phi}=1$ for every $n \in \mathbb{N}$. By the assumed condition $\delta_{2}$ on $\Phi$, we have $l_{\Phi}\left(x_{n}\right)=1, n \in \mathbb{N}$. Let $b_{i}$ be such that $\phi_{i}^{*}\left(b_{i}\right)=\delta$, where $\delta$ is a constant in condition (S). It follows that $\phi_{i}^{*}\left(\lambda b_{i}\right) \leq A_{j} \delta+c_{i j}$ for all $j \in \mathbb{N}$ and $n \geq n_{j}$. Given $\epsilon>0$, fix $j$ with $A_{j}<\epsilon$ and then choose $n_{0} \geq n_{j}$ such that $c_{i j}<\epsilon$ for all $i \geq n_{0}$. Thus $\phi_{i}^{*}\left(\lambda b_{i}\right) \leq \epsilon(\delta+1)$ for all $i>n_{0}$, and $\lim _{i} \phi_{i}^{*}\left(\lambda b_{i}\right)=0$. By Lemma 4.6, $\inf _{i} b_{i}=b>0$ and thus $0=\lim _{i} \phi_{i}^{*}\left(\lambda b_{i}\right) \geq \lim _{i} \phi_{i}^{*}(\lambda b)$, which implies that $\lim _{i} \phi_{i}^{*}(a)=0$ where $a=\lambda b$.

Define now for every $n \in \mathbb{N}, y_{n}(i)=\phi_{i}^{\prime}\left(x_{n}(i)\right), i \in S_{n}$. Let's choose $0<A<$ $\min (\beta, 1, \delta / K)$, where $\beta, K$ are constants in condition $\delta_{2}$, and $\delta$ is a constant in (S). By Lemma 4.6, we obtain that $\left\|x_{n}\right\|_{\ell_{\infty}} \rightarrow 0$. This implies that there exists $n_{A} \in \mathbb{N}$ such that for all $n>n_{A}$ and all $n \in \mathbb{N}, x_{n}(i) \leq A$, and hence $\phi_{i}\left(x_{n}(i)\right) \leq A \phi_{i}(1)=A \leq \beta$. Let $i_{A}$ be such that $c_{i}<A$ for $i \geq i_{A}$. It follows by applying the Young's equality that for every $n \geq n_{A}$ and $i \geq i_{A}$,

$$
\begin{aligned}
\phi_{i}^{*}\left(\phi_{i}^{\prime}\left(x_{n}(i)\right)\right) & =x_{n}(i) \phi_{i}^{\prime}\left(x_{n}(i)\right)-\phi_{i}\left(x_{n}(i)\right) \leq \phi_{i}\left(2 x_{n}(i)\right)-\phi_{i}\left(x_{n}(i)\right) \\
& \leq K \phi_{i}\left(x_{n}(i)\right)+c_{i}-\phi_{i}\left(x_{n}(i)\right) \leq(K-1) A+A \leq \delta .
\end{aligned}
$$

Now modifying $x_{n}$ if necessary we assume that $\phi_{i}^{*}\left(y_{n}(i)\right) \leq \delta$ and $x_{n}(i) \leq A$ for every $i, n \in \mathbb{N}$. Applying then condition (S), $\phi_{i}^{*}\left(\lambda y_{n}(i)\right) \leq A_{j} \phi_{i}^{*}\left(y_{n}(i)\right)+c_{i j}$ for all $i \geq n_{j}$. Since $\min S_{n_{j}} \geq n_{j}$,

$$
\phi_{i}^{*}\left(\lambda y_{n_{j}}(i)\right)=\phi_{i}^{*}\left(\lambda \phi_{i}^{\prime}\left(x_{n_{j}}(i)\right)\right) \leq A_{j} \phi_{i}^{*}\left(\phi_{i}^{\prime}\left(x_{n_{j}}(i)\right)\right)+c_{i j} .
$$


Thus for every $j \in \mathbb{N}$,

$$
\begin{aligned}
\sum_{i \in S_{n_{j}}} \phi_{i}^{*}\left(\lambda y_{n_{j}}(i)\right) & \leq A_{j} \sum_{i \in S_{n_{j}}} \phi_{i}^{*}\left(\phi_{i}^{\prime}\left(x_{n_{j}}(i)\right)\right)+\sum_{i \in S_{n_{j}}} c_{i j} \\
& \leq A_{j} \sum_{i \in S_{n_{j}}}\left(\phi_{i}\left(2 x_{n_{j}}(i)-\phi_{i}\left(x_{n_{j}}(i)\right)\right)+\sum_{i \geq n_{j}} c_{i j}\right. \\
& \leq A_{j} \sum_{i \in S_{n_{j}}}\left((K-1) \phi_{i}\left(x_{n_{j}}(i)\right)+c_{i}\right)+\sum_{i \geq n_{j}} c_{i j} \\
& \leq A_{j}(K-1) I_{\Phi}\left(x_{n_{j}}\right)+A_{j} \sum_{i=1}^{\infty} c_{i}+\sum_{i \geq n_{j}} c_{i j} \leq M A_{j}+\sum_{i \geq n_{j}} c_{i j}
\end{aligned}
$$

where $M=K-1+\sum_{i=1}^{\infty} c_{i}$. Therefore

$$
\sum_{j=1}^{\infty} \sum_{i \in S_{n_{j}}} \phi_{i}^{*}\left(\lambda y_{n_{j}}(i)\right) \leq M \sum_{j=1}^{\infty} A_{j}+\sum_{j=1}^{\infty} \sum_{i=n_{j}}^{\infty} c_{i j}<\infty .
$$

Defining $y(i)=y_{n_{j}}(i)$ for $i \in S_{n_{j}}, j \in \mathbb{N}$, and $y(i)=0$ otherwise, we obtain that $I_{\Phi^{*}}(\lambda y)<\infty$, and so $y \in \ell_{\Phi^{*}}$. However, for every $j \in \mathbb{N}$,

$$
\sum_{i=1}^{\infty} y(i) x_{n_{j}}(i) \geq \sum_{i \in S_{n_{j}}} x_{n_{j}}(i) \geq \sum_{i \in S_{n_{j}}} \phi_{i}\left(x_{n_{j}}(i)\right)=I_{\Phi}\left(x_{n_{j}}\right)=1,
$$

which yields that $\left(x_{n}\right)$ does not converge to zero weakly in $\ell_{\Phi}$. This contradiction completes the proof.

Our next result also states sufficient conditions for the Schur property of $\ell_{\Phi}$. Although it may be less general than the previous one (see Remark after Theorem 5.1), it has some advantages. The conditions are stated directly in terms of $\phi_{n}$ and are more convenient for verification in particular situations.

In what follows for a given finite valued and vanishing only at zero Orlicz function $\phi$ we define on $[0, \infty)$ a function $\hat{\phi}$ by

$$
\hat{\phi}(t)=\inf _{0<u \leq 1} \frac{\phi(t u)}{\phi(u)}, \quad t \geq 0 .
$$

We recall that Orlicz functions $\phi$ and $\psi$ are equivalent on $A \subset \mathbb{R}_{+}(\phi \asymp \psi$ on $A$ ), whenever $\phi\left(C_{1} u\right) \leq \psi(u) \leq \phi\left(C_{2} u\right)$ for all $u \in A$.

THEOREM 4.8. Let $\Phi=\left(\phi_{n}\right)$ be a normalized Musielak-Orlicz function such that all $\phi_{n}$ are finite and vanish only at zero. Assume that one of the following conditions holds true. 
(i) $\Phi \in\left(\delta_{2}\right), \lim _{n} \phi_{n}^{*}(a)=0$ for some $a>0$ and for any positive sequence $\left(\alpha_{n}\right)$ in the unit ball of $c_{0}$ a sequence $\left(\phi_{n}\left(\alpha_{n} t\right) / \phi_{n}\left(\alpha_{n}\right)\right)$ converges uniformly to $\phi(t) \asymp t$ on $[0,1]$.

(ii) $\Phi \in\left(\delta_{2}\right)$ and for any positive sequence $\left(\alpha_{n}\right)$ in the unit ball of $\ell_{\infty}$ a sequence $\left(\phi_{n}\left(\alpha_{n} t\right) / \phi_{n}\left(\alpha_{n}\right)\right)$ converges uniformly to $\phi(t) \asymp t$ on $[0,1]$.

(iii) $\left(\hat{\phi}_{n}\right)$ converges uniformly to $t$ on $[0,1]$.

Then Musielak-Orlicz space $\ell_{\Phi}$ has the Schur property.

PROOF. We shall apply here Theorem 4.4. Assume at first that (i) is satisfied. Let then $\left(\psi_{i}(t)\right)=\left(\sum_{j=n_{i}+1}^{n_{i+1}} \phi_{j}\left(\alpha_{j} t\right)\right)$ be any $\Phi$-convex $c_{0}$-block of $\left(\phi_{n}\right)$. Since $\left(\alpha_{j}\right) \in c_{0}^{+}$ and $\sum_{j=n_{i}+1}^{n_{i+1}} \phi_{j}\left(\alpha_{j}\right)=1$, without loss of generality we may assume that $\alpha_{j}>0$ for all $j \in \mathbb{N}$. In view of the assumption $\sup _{0 \leq t \leq 1}\left|\phi_{n}\left(\alpha_{n} t\right) / \phi_{n}\left(\alpha_{n}\right)-\phi(t)\right| \rightarrow 0$, we have

$$
\begin{aligned}
\sup _{0 \leq t \leq 1}\left|\psi_{i}(t)-\phi(t)\right| & =\sup _{0 \leq t \leq 1}\left|\sum_{j=n_{i}+1}^{n_{i+1}}\left(\phi_{j}\left(t \alpha_{j}\right)-\phi(t) \phi_{j}\left(\alpha_{j}\right)\right)\right| \\
& \leq \sup _{0 \leq t \leq 1} \sum_{j=n_{i}+1}^{n_{i+1}}\left|\left(\phi(t)-\phi_{j}\left(\alpha_{j} t\right) / \phi_{j}\left(\alpha_{j}\right)\right) \phi_{j}\left(\alpha_{j}\right)\right| \\
& \leq \max _{n_{i}+1 \leq j \leq n_{i+1}} \sup _{0 \leq t \leq 1}\left|\phi(t)-\phi_{j}\left(\alpha_{j} t\right) / \phi_{j}\left(\alpha_{j}\right)\right| \rightarrow 0
\end{aligned}
$$

as $i \rightarrow \infty$. Thus $\psi_{i}(t) \rightarrow \phi(t)$ uniformly on $[0,1]$. Given $\epsilon>0$, there exists a subsequence $\left(i_{k}\right)$ such that for every $t \in[0,1]\left|\psi_{i_{k}}(t)-\phi(t)\right| \leq \epsilon / 2^{k}, k \in \mathbb{N}$. This easily implies that $\ell_{\left(\psi_{\left.i_{k}\right)}\right.}=\ell_{\phi}$, or equivalently that $\left(\psi_{i_{k}}\right)_{k=1}^{\infty}$ is equivalent to $\phi$ and hence to a linear function, by $\phi(t) \asymp t$ on $[0,1]$.

If (ii) holds, the proof is similar. In order to prove (iii) observe that if $\hat{\phi}_{n}(t)$ converges uniformly to $\phi(t) \asymp t$ on [0,1], then for sufficiently large $n \in \mathbb{N}$ and some $\epsilon>0$ we have $(t-\epsilon) \phi_{n}(u) \leq \phi_{n}(t u)$ for $t, u \in[0,1]$. This clearly implies that $\Phi \in\left(\delta_{2}\right)$. Moreover,

$$
\sup _{0 \leq t \leq 1}\left|t-\frac{\phi_{j}\left(\alpha_{j} t\right)}{\phi_{j}\left(\alpha_{j}\right)}\right| \leq \sup _{0 \leq t \leq 1}\left|t-\hat{\phi}_{n}(t)\right| \rightarrow 0
$$

for any positive sequence $\left(\alpha_{j}\right)$ in the unit ball of $\ell_{\infty}$. This shows that the condition (iii) implies (ii), and thus $\ell_{\Phi} \in(\mathrm{SP})$.

Below we provide some necessary conditions for the Schur property of $\ell_{\Phi}$.

PROPOSITION 4.9. Let $\Phi=\left(\phi_{n}\right)$ be an arbitary Musielak-Orlicz function. If $\ell_{\Phi}$ has the Schur property then $\Phi \in\left(\delta_{2}\right)$ and $\alpha(\Phi)=1$. 
PROOF. If $\Phi \notin\left(\delta_{2}\right)$, then $\ell_{\Phi}$ contains an isomorphic copy of $\ell_{\infty}$ and so $\ell_{\Phi} \notin$ (SP). If $\alpha(\Phi)>1$ then $\beta\left(\Phi^{*}\right)<\infty$, and hence $\Phi^{*} \in\left(\delta_{2}\right)$ (see [17]). But then $\ell_{\Phi}$ is reflexive (both $\Phi$ and $\Phi^{*}$ satisfy condition $\delta_{2}$ ), which is impossible since $\ell_{\Phi} \in$ (SP).

PROPOSITION 4.10. Let $\Phi=\left(\phi_{n}\right)$ be a nondecreasing sequence of normalized Orlicz functions. If $\ell_{\Phi}$ has the Schur property then there exists an Orlicz function $\phi$ such that $\phi_{n}(u) \rightarrow \phi(u)$ uniformly for $u \in[0,1]$ and $\phi(u) \asymp u$.

PROOF. Since $\phi_{n}(u) \leq 1$ for all $u \in[0,1], n \in \mathbb{N}$, and $\left(\phi_{n}\right)$ is a monotone sequence of functions, by the Dini's theorem there exists a function $\phi$ such that $\phi_{n}(u) \rightarrow \phi(u)$ uniformly on $[0,1]$. By Theorem 4.4, there exist $\left(n_{k}\right) \subset \mathbb{N}, C>0$ and $\left(a_{k}\right) \in \ell_{1}^{+}$such that $C u \leq \phi_{n_{k}}(u) \leq u$ for all $k \in \mathbb{N}$ and $a_{k} \leq u \leq 1$. It follows that for all $u \geq 0$, $C u \leq \phi(u) \leq u$, that is, $\phi(u) \asymp u$.

The Yamamuro characterization of the Schur property of $\ell_{\Phi}$ is a corollary from our results.

COROLLARY 4.11 (see [36]). Let $\Phi=\left(\phi_{n}\right)$ be a Musielak-Orlicz function such that each $\phi_{n}$ is finite, vanishes only at zero and $\phi_{n}^{\prime}$ exists for all $n \in \mathbb{N}$. If $\ell_{\Phi}$ has the Schur property then $\lim _{n} a\left(\phi_{n}\right)=1$. If $\lim _{n} b\left(\phi_{n}\right)=1$ then $\ell_{\Phi}$ has the Schur property.

PROOF. The necessity part is a consequence of Proposition 4.9. In fact, by definition of the Simonenko index $a_{n}=a\left(\phi_{n}\right)$, it is easy to check that the function $t \mapsto \phi_{n}(t) / t^{a_{n}}$ is increasing for $t>0$, and hence $\phi_{n}(\lambda t) \leq \lambda^{a_{n}} \phi_{n}(t) \leq \lambda^{\text {inf }_{n} a_{n}} \phi_{n}(t)$ for all $\lambda \geq 1$, $n \in \mathbb{N}$ which simply yields that $\inf _{n} a_{n} \leq \alpha(\Phi)$. But $\alpha(\Phi)=1$ whenever $\ell_{\Phi} \in(\mathrm{SP})$, by Proposition 4.9, and hence $\lim _{n} a_{n}=1$.

Applying at first Theorem 4.8 (ii), we shall show the sufficiency part. Observe that if there exists a real sequence $\left(b_{n}\right)$ such that $b_{n} \rightarrow 1$ as $n \rightarrow \infty$ and each function $t \mapsto \phi_{n}(t) / t^{b_{n}}$ is non-increasing on $(0,1]$, then $\ell_{\Phi} \in(\mathrm{SP})$. Indeed, for any $t, u \in(0,1], \phi_{n}(t u) / \phi_{n}(u) \geq t^{b_{n}}$ and so $\hat{\phi}_{n}(t) \geq t^{b_{n}}$. Hence $0 \leq t-\hat{\phi}_{n}(t) \leq t-t^{b_{n}}$ for $t \in[0,1]$, and since $\sup _{t \in[0,1]}\left|t-t^{b_{n}}\right| \rightarrow 0, \hat{\phi}_{n}(t) \rightarrow t$ uniformly on $(0,1]$. Thus, by Theorem 4.8, $\ell_{\Phi} \in$ (SP). Now letting $b_{n}=\sup _{0<t \leq 1}\left(t \phi_{n}^{\prime}(t) / \phi_{n}(t)\right)$, it is easy to check that the functions $t \mapsto \phi_{n}(t) / t^{b_{n}}$ are decreasing on $(0,1]$. Thus if we assume that $b\left(\phi_{n}\right) \rightarrow 1$, then $1<b_{n} \leq b\left(\phi_{n}\right) \rightarrow 1$, and so $\ell_{\Phi} \in(\mathrm{SP})$.

REMARK. Note that the sufficiency part in the above result also follows from Theorem 4.7. Indeed, if $b\left(\phi_{n}\right) \rightarrow 1$ then $a\left(\phi_{n}^{*}\right) \rightarrow \infty$, in view of the relation $1 / b\left(\phi_{n}\right)+1 / a\left(\phi_{n}^{*}\right)=1$. Moreover, the functions $t \mapsto \phi_{n}^{*}(t) / t^{a\left(\phi_{n}^{*}\right)}$ are increasing for $t>0$, and so for any $0<\lambda<1$, $\sup _{t>0}\left(\phi_{n}^{*}(\lambda t) / \phi_{n}^{*}(t)\right) \leq \lambda^{a\left(\phi_{n}^{*}\right)}, n \in \mathbb{N}$, which yields $\lim _{n} \sup _{t>0}\left(\phi_{n}^{*}(\lambda t) / \phi_{n}^{*}(t)\right)=0$ and thus $\Phi^{*} \in(S)$, and by Theorem 4.7, $\ell_{\Phi} \in(\mathrm{SP})$. 


\section{Schur property in weighted Orlicz sequence spaces}

Important class of Musielak-Orlicz sequence spaces is a class of the weighted Orlicz sequence spaces $\ell_{\phi}(w)$ studied among others in [29]. Given an Orlicz function $\phi$ and a weight sequence $w=\left(w_{n}\right)$, that is, a real positive sequence, let $\Phi=\left(\phi_{n}\right)$, where $\phi_{n}(u)=\phi(u) w_{n}$ for each $n \in \mathbb{N}$. Then $\ell_{\phi}(w)=\ell_{\Phi}$ is called a weighted Orlicz space.

Recall that an Orlicz function $\phi$ satisfies condition $\Delta_{2}\left(\phi \in \Delta_{2}\right)$ if $\phi$ satisfies condition $\Delta_{2}$ at infinity, that is, $\lim \sup _{u \rightarrow \infty} \phi(2 u) / \phi(u)<\infty$, and if it satisfies condition $\Delta_{2}$ at zero, that is, $\lim \sup _{u \rightarrow 0^{+}} \phi(2 u) / \phi(u)<\infty$.

In what follows we say that a weight sequence $w=\left(w_{n}\right)$ belongs to class $\Lambda_{1}$ ( $\left.w \in \Lambda_{1}\right)$ or $\Lambda_{\infty}\left(w \in \Lambda_{\infty}\right)$ whenever $w \in \ell_{1}$ or $w_{n} \rightarrow \infty$, respectively. Recall also, following [29, Definition 2.6], that a weight sequence $w=\left(w_{n}\right)$ belongs to class $\Lambda$ $(w \in \Lambda)$ if there is a subsequence $\left(w_{n_{k}}\right)$ of $\left(w_{n}\right)$ such that

$$
\lim _{k \rightarrow \infty} w_{n_{k}}=0 \text { and } \sum_{k=1}^{\infty} w_{n_{k}}=\infty .
$$

We will present further some applications of the obtained results in Musielak-Orlicz spaces to weighted sequence Orlicz spaces. The next result, showed first by Wnuk [33] as a corollary of characterization of Orlicz spaces $L_{\phi}(0,1)$ with the positive Schur property [20], is also a consequence of Theorem 4.7.

THEOREM 5.1. Let $\Phi=\left(\phi_{n}\right)$ be such that $\phi_{n}(u)=\phi(u) w_{n}$, where $\phi$ is a finite valued Orlicz function with $\lim _{u \rightarrow \infty} \phi(u) / u=\infty$, and $w \in \Lambda_{1}$. If $\phi$ satisfies condition $\Delta_{2}$ at infinity and $\lim _{u \rightarrow \infty} \phi^{*}(a u) / \phi^{*}(u)=\infty$ for some $a>1$, then $\ell_{\Phi}$ has the Schur property.

PROOF. By the assumption of $\Delta_{2}$ condition at infinity of $\phi$, it is easy to show that $\Phi \in\left(\delta_{2}\right)$. Let now $\Psi=\left(\psi_{n}\right)$, where $\psi_{n}(u)=\phi\left(a_{n} u\right) w_{n}$, and $a_{n}=\phi^{-1}\left(1 / w_{n}\right)$. Then $\ell_{\Psi}$ is isometrically isomorphic to $\ell_{\Phi}$ and $\psi_{n}(1)=1, n \in \mathbb{N}$, and

$$
\psi_{n}^{*}(u)=w_{n} \phi^{*}\left(u / a_{n} w_{n}\right)
$$

are finite valued by the assumption $\lim _{u \rightarrow \infty} \phi(u) / u=\infty$. In view of Theorem 4.7, we complete the proof if we show that $\Psi^{*} \in(S)$.

Let $\delta>0$ be any number and $b_{i}$ be such that $\psi_{i}^{*}\left(b_{i}\right)=\delta$. By Lemma 4.6, $b=\sup _{i} b_{i}<\infty$. Let further $\lambda=1 / a$ and $A_{j}=1 / a^{j}$. By the assumption on $\phi^{*}$, there exists $u_{j} \geq 0$ such that for $u \geq u_{j} \phi^{*}(u / a)-\left(1 / a^{j}\right) \phi^{*}(u) \leq 0$. Observe also that in view of $\lim _{u \rightarrow \infty} \phi(u) / u=\infty, \lim _{i \rightarrow \infty} b /\left(a_{i} w_{i}\right)=\infty$ and so

$$
\frac{b}{a_{i} w_{i}} \geq u_{j}
$$


for all $i \geq m_{j}$ and some $m_{j} \in \mathbb{N}$. Hence for all $i \geq m_{j}$

$$
\begin{aligned}
\sup _{\psi_{i}^{*}(u) \leq \delta}\left\{\psi_{i}^{*}(\lambda u)-A_{j} \psi_{i}^{*}(u)\right\} & \leq w_{i} \sup _{0 \leq u \leq b}\left\{\phi^{*}\left(\frac{u}{a a_{i} w_{i}}\right)-\frac{1}{a^{j}} \phi^{*}\left(\frac{u}{a_{i} w_{i}}\right)\right\} \\
& =w_{i} \sup _{0 \leq u \leq b / a_{i} w_{i}}\left\{\phi^{*}\left(\frac{u}{a}\right)-\frac{1}{a^{j}} \phi^{*}(u)\right\}=w_{i} B_{j},
\end{aligned}
$$

where $B_{j}=\sup _{0 \leq u \leq u_{j}}\left\{\phi^{*}(u / a)-\phi^{*}(u) / a^{j}\right\}$. Choosing $n_{j} \geq m_{j}$ such that

$$
\sum_{i \geq n_{j}}^{\infty} w_{i} B_{j} \leq A_{j}
$$

we finally obtain that $\sum_{j=1}^{\infty} \sum_{i \geq n_{j}} w_{i} B_{j} \leq \sum_{j=1}^{\infty} A_{j}<\infty$, which shows condition (S) for $\Psi^{*}$.

REMARK. Examples of Orlicz functions $\phi$ satisfying the conditions (for any $a>1$ ) of the above theorem are, for instance, the following: $\phi(t)=t \ln (1+t) ; \phi(t)=$ $(1+t) \ln (1+t)-t$ for $t \geq 0$. Thus the weighted Orlicz sequence spaces $\ell_{\phi}(w)$ has the Schur property for any weight sequence $w \in \Lambda_{1}$. Note that this property can not be concluded from Theorem 4.8 and hence from the Yamamuro's result as well. To see this consider only $\phi(t)=t \ln (1+t)$. Let $\Phi=\left(\phi_{n}\right)$, where $\phi_{n}(t)=\phi\left(a_{n} t\right) w_{n}$ with $a_{n}=\phi^{-1}\left(1 / w_{n}\right)$ and $w \in \Lambda_{1}$. It is clear that $\Phi$ is a normalized Musielak-Orlicz function and $\ell_{\Phi}$ is isometrically isomorphic to $\ell_{\phi}\left(w_{n}\right)$ and so $\ell_{\Phi} \in$ (SP). However, if we take $\alpha_{n}=1 / a_{n}$ for every $n \in \mathbb{N}$, we have $\alpha_{n} \rightarrow 0$ and

$$
\frac{\phi_{n}\left(\alpha_{n} t\right)}{\phi_{n}\left(\alpha_{n}\right)}=\phi(t) / \ln 2
$$

for every $n \in \mathbb{N}, t \geq 0$. Since $\phi(t) \asymp t^{2}$ on $[0,1]$, so Theorem 4.8 does not work in this case.

In [10] it is proved that for every separable Musielak-Orlicz sequence space $\ell_{\Phi}$ there exists an Orlicz function $\phi$ and a weight sequence $w=\left(w_{n}\right) \in \Lambda_{\infty}$ such that the weighted Orlicz sequence space $\ell_{\phi}(w)$ is (order) isomorphic to $\ell_{\Phi}$. This result seems to explain that in general for a given $w \in \Lambda_{\infty}$, the problem of characterization of the Schur property in $\ell_{\phi}(w)$ in terms of $\phi$ is nontrivial.

Following [23] (see also [9, 27]), for a given finite valued Orlicz function $\phi$ vanishing only at 0 and for any $0<s \leq \infty$, we define several subsets of $C(0,1)$ as follows:

$$
E_{\phi, s}=\overline{\left\{\frac{\phi(t u)}{\phi(t)}: 0<t<s\right\}}, \quad E_{\phi, s}^{\infty}=\overline{\left\{\frac{\phi(t u)}{\phi(t)}: s \leq t\right\}}
$$


$E_{\phi}^{0}=\bigcap_{0<s \leq 1} E_{\phi, s}, E_{\phi}^{\infty}=\bigcap_{s \geq 1} E_{\phi, s}^{\infty}, C_{\phi}^{\infty}=\overline{\operatorname{conv}} E_{\phi}^{\infty}$, and $E_{\phi}(0, \infty)=E_{\phi, \infty}$, $C_{\phi}(0, \infty)=\overline{c o n v} E_{\phi}(0, \infty)$. They are all compact subsets of $C(0,1 / 2)$, and if $\phi \in \Delta_{2}$ at zero then $E_{\phi, s}$ and $E_{\phi}^{0}$ are compact subsets of $C(0,1)$, if $\phi \in \Delta_{2}$ at infinity, then $E_{\phi, s}^{\infty}, E_{\phi}^{\infty}$ and $C_{\phi}^{\infty}$ are compact in $C(0,1)$, and finally if $\phi \in \Delta_{2}$, then $E_{\phi}(0, \infty)$ and $C_{\phi}(0, \infty)$ are also compact in $C(0,1)$.

PROPOSITION 5.2. Let $\phi$ be a finite valued Orlicz function which vanishes only at zero. Then the following statements hold true.

(i) For every $\psi \in E_{\phi}^{\infty}$ there exists a weight sequence $w \in \Lambda_{1}$ such that $\ell_{\phi}(w)$ is isomorphic to an Orlicz sequence space $\ell_{\psi}$.

(ii) For every $\psi \in E_{\phi}^{0}$ there exists a weight sequence $w \in \Lambda_{\infty}$ such that $\ell_{\phi}(w)$ is isomorphic to an Orlicz sequence space $\ell_{\psi}$.

(iii) If $\ell_{\phi}(w)$ has the Schur property for every $w \in \Lambda_{1}$ (respectively, $w \in \Lambda_{\infty}$ ), then $\psi(t) \asymp t$ on $[0,1]$ for every $\psi \in E_{\phi}^{\infty}$ (respectively, $\psi \in E_{\phi}^{0}$ ).

(iv) If $\phi$ satisfies condition $\Delta_{2}, \lim _{u \rightarrow \infty} \phi(u) / u=\infty$ and $\lim _{u \rightarrow \infty} \phi^{*}(a u) / \phi^{*}(u)=$ $\infty$ for some $a>1$, then $\psi(t) \asymp t$ on $[0,1]$ for any $\psi \in E_{\phi}^{\infty}$.

ProOF. The proof of (ii) is similar to the proof of (i), which is presented in [9, Proposition 1]. (iii) is an immediate consequence of (i) and (ii) since $\ell_{\psi} \in$ (SP) if and only if $\psi(t) \asymp t$ on $[0,1]$. Finally, (iv) follows by (iii) and Theorem 5.1.

Next we present a characterization of (SP) in the weighted Orlicz sequence spaces $\ell_{\phi}(w)$ in the case whenever the weight sequence is in the class $\Lambda$. Note that it is shown in [7] that given $w \in \Lambda$ for every positive sequence $v=\left(v_{k}\right)$ the canonical basis of $\ell_{\phi}(v)$ is equivalent to a block basic sequence with constant coefficients of the canonical basis of $\ell_{\phi}(w)$. In fact by the result [3], it follows that the series $\sum_{n=1}^{\infty} w_{n}$ has the Darboux property for every $w \in \Lambda$, that is, for every positive real number $x$ there exists a subseries $\sum_{j=1}^{\infty} w_{k_{n j}}$ which converges to $x$. An immediate consequence of this result is the following simple observation, which will be used in the sequel, that for any weight sequence $v=\left(v_{k}\right)$ there exists a sequence $\left(I_{k}\right)$ of pairwise disjoint subsets of $\mathbb{N}$ such that $\sum_{n \in I_{k}} w_{n}=v_{k}$ for every $k \in \mathbb{N}$. This easily yields that the map $\left(x_{n}\right) \mapsto \sum_{n} x_{n} \chi_{I_{n}}$ is an order isometry of the weighted Orlicz sequence space $\ell_{\phi}(v)$ onto a sublattice of $\ell_{\phi}(w)$.

THEOREM 5.3. Let a weight sequence $w=\left(w_{n}\right) \in \Lambda$ and let $\phi$ be a finite valued Orlicz function which vanishes only at zero. Then the following statements are equivalent:

(i) $\ell_{\phi}(w)$ has the Schur property;

(ii) $\phi \in \Delta_{2}$ and $\psi(t) \asymp t$ on $[0,1]$ for every $\psi \in C_{\phi}(0, \infty)$;

(iii) $\phi \in \Delta_{2}, \phi(t) \asymp t$ on $[0,1]$ and $\psi(t) \asymp t$ on $[0,1]$ for every $\psi \in C_{\phi}^{\infty}$. 
Proof. (i) implies (ii). Let $\Phi=\left(\phi_{n}\right)$ with $\phi_{n}(t)=\phi(t) w_{n}$. First note that $\Phi \in\left(\delta_{2}\right)$, otherwise $\ell_{\Phi}$ contains an isomorphic copy of $\ell_{\infty}$ and so $\ell_{\phi}(w) \notin(\mathrm{SP})$. It is clear that $\Phi \in\left(\delta_{2}\right)$ implies $\phi \in \Delta_{2}$ (see also [17]).

We shall show now that every function in $C_{\phi}(0, \infty)$ is equivalent to a linear function on $[0,1]$. Indeed, let $\psi \in C_{\phi}(0, \infty)$. Then there exist a sequence $\left(I_{i}\right)_{i=1}^{\infty}$ of finite subsets of $\mathbb{N}$ and positive sequences of real numbers $\left(\alpha_{j}^{i}\right)$ and $\left(s_{j}^{i}\right)$ such that $\psi_{i} \rightarrow \psi$ uniformly on $[0,1]$, where $\psi_{i}(t)=\sum_{j \in I_{i}} \alpha_{j}^{i} \phi\left(s_{j}^{i} t\right) / \phi\left(s_{j}^{i}\right)$ for $t \geq 0$ with $\psi_{i}(1)=1$ for all $i \in \mathbb{N}$. Without loss of generality we assume that $\lim _{n} w_{n}=0$ and $\sum_{n=1}^{\infty} w_{n}=\infty$. By the Darboux property of the series $\sum_{n=1}^{\infty} w_{n}$, there exists a sequence $(I(i, j))_{i \in \mathbb{N}, j \in A_{i}}$ of pairwise disjoint subsets of $\mathbb{N}$ such that for every $i \in \mathbb{N}$ and $j \in I_{i}$ it holds $\sum_{n \in I(i, j)} w_{n}=\alpha_{j}^{i} / \phi\left(s_{j}^{i}\right)$. If we define $\Psi=\left(\psi_{i}\right)$, then for any $\left(x_{i}\right) \in \ell_{\Psi}$, we have

$$
\sum_{i=1}^{\infty} \psi_{i}\left(\left|x_{i}\right|\right)=\sum_{i=1}^{\infty} \sum_{j \in I_{i}} \sum_{n \in I(i, j)} \phi\left(s_{j}^{i}\left|x_{i}\right|\right) w_{n}=\sum_{n=1}^{\infty} \phi\left(\sum_{i=1}^{\infty} \sum_{j \in I_{i}} s_{j}^{i}\left|x_{i}\right| \chi_{I(i, j)}(n)\right) w_{n} .
$$

This shows that the map $\left(x_{n}\right) \mapsto\left(\sum_{i=1}^{\infty} \sum_{j \in I_{i}} x_{i} s_{j}^{i} \chi_{I(i, j)}(n)\right)_{n=1}^{\infty}$ is an order isometry of $\ell_{\Psi}$ into a sublattice of $\ell_{\phi}(w)$. Hence $\ell_{\Psi}$ has the Schur property by $\ell_{\phi}(w) \in(\mathrm{SP})$. Since $\left(\psi_{i}\right)$ converges uniformly to $\psi$ on $[0,1]$, there is a subsequence $\left(\psi_{i_{k}}\right)$ of $\left(\psi_{i}\right)$ such that the canonical basis $\left(e_{k}\right)$ of an Orlicz space $\ell_{\psi}$ and $\left(e_{k}\right)$ of $\ell_{\left(\psi_{i_{k}}\right)}$ are equivalent. In consequence, we conclude that $\ell_{\psi}$ has the Schur property and thus $\psi(t) \asymp t$ on $[0,1]$.

(ii) implies (iii). Since $C_{\phi}^{\infty} \subset C_{\phi}(0, \infty)$ and $\phi \in C_{\phi}(0, \infty)$, the implication is clear.

(iii) implies (i). First we show that (iii) implies that $\psi(t) \asymp t$ on $[0,1]$ for any $\psi \in C_{\phi}(0, \infty)$. To see this take $\psi \in C_{\phi}(0, \infty)$. Using the Krein-Milman Theorem, we conclude that (see [27, proof of Theorem 1.1]) $\psi$ can be written as a convex combination: $\psi=\lambda_{1} \psi_{1}+\lambda_{2} \psi_{2}+\lambda_{3} \psi_{3}$ with $\psi_{1} \in \overline{\operatorname{conv}} E_{\phi, 1}, \psi_{2} \in C_{\phi}^{\infty}$ and with $\psi_{3}$ having a representation

$$
\psi_{3}(t)=\int_{1}^{\infty} \frac{\phi(t s)}{\phi(s)} d v(s)
$$

where $v$ is a probability measure on $[1, \infty)$ such that $v(\{1\})=0$. This implies that $C \phi(t) \leq \psi_{3}(t) \leq t$ for all $t \in[0,1]$, where $0<C=\int_{1}^{\infty}(1 / \phi(s)) d \nu(s)<\infty$. Since $\phi(t) \asymp t$ on $[0,1]$, we get $\psi_{3}(t) \asymp t$ on $[0,1]$. Clearly, $\phi(t) \asymp t$ on $[0,1]$ implies that any function in $\overline{c o n v} E_{\phi .1}$ is equivalent to a linear function on $[0,1]$. In consequence, the equality $\psi=\lambda_{1} \psi_{1}+\lambda_{2} \psi_{2}+\lambda_{3} \psi_{3}$ implies the required equivalence $\psi(t) \asymp t$ on $[0,1]$.

In order to finish the proof we use Theorem 4.4. Let $\left(\psi_{i}\right)$ be any $\Phi$-convex block of $\left(\phi_{n}\right)$, that is, $\psi_{i}(t)=\sum_{j=n_{i}+1}^{n_{i+1}} \phi_{j}\left(t \alpha_{j}\right)$ with $\sum_{j=n_{i}+1}^{n_{i+1}} \phi_{j}\left(\alpha_{j}\right)=1$. This implies that

$$
\psi_{i}(t)=\sum_{j=n_{i}+1}^{n_{i+1}} \phi_{j}\left(\alpha_{j}\right) \frac{\phi\left(t \alpha_{j}\right)}{\phi\left(\alpha_{j}\right)}
$$


that is, $\left(\psi_{i}\right) \subset C_{\phi}(0, \infty)$. By the compactness argument $\left(\psi_{i}\right)$ contains a subsequence $\left(\psi_{i_{k}}\right)$ which converges uniformly to some $\psi \in C_{\phi}(0, \infty)$. However, we proved that $\psi(t) \asymp t$ on $[0,1]$ for any $\psi \in C_{\phi}(0, \infty)$, and this finishes the proof.

COROLlaRY 5.4. Let $w=\left(w_{n}\right) \in \Lambda$ and let $\phi(t) \asymp \max \{t, \varphi(t)\}$ on $\mathbb{R}_{+}$, where $\varphi$ is a finite valued and differentiable at infinity Orlicz function satisfying condition $\Delta_{2}$ at infinity and $\lim _{t \rightarrow \infty}\left(t \varphi^{\prime}(t) / \varphi(t)\right)=p$. Then $\ell_{\phi}(w)$ has the Schur property if and only if $p=1$.

Proof. It follows from [9, Proposition 6] that $E_{\varphi}^{\infty}=\left\{t^{p}\right\}$. This implies that also $C_{\varphi}^{\infty}=\left\{t^{p}\right\}$. Clearly, $\phi$ satisfies condition $\Delta_{2}$ and $\phi(t) \asymp t$ on $[0,1]$. Thus Theorem 5.3 applies.

EXAMPLE. Let $\ell_{\phi}(w)$ be a weighted Orlicz sequence space with $w \in \Lambda$ and $\phi(t) \asymp$ $\max \{t, \varphi(t)\}$ on $\mathbb{R}_{+}$, where $\varphi$ is the following function

$$
\varphi(t)=t^{p} \ln ^{\alpha}(e+t) \text { for } t \geq 0,1 \leq p<\infty, \alpha \in \mathbb{R} .
$$

Then by Corollary 5.4, $\ell_{\phi}(w)$ has the Schur property if and only if $p=1$, since $\lim _{t \rightarrow \infty} t \varphi^{\prime}(t) / \varphi(t)=p$.

Combining the above example with the following result below, we conclude that there exist weighted Orlicz sequence spaces with the Schur property which are not isomorphic to any $\mathscr{L}_{1}$-space (in the sense of [22]) and in particular, it is not isomorphic to any AL-space (see [2]). In consequence, it follows by Theorem 3.5, that these spaces are not isomorphic to any symmetric space.

Proposition 5.5. If $w \in \Lambda$ and $\phi \in \Delta_{2}$ with $\lim _{u \rightarrow \infty} \phi(u) / u=\infty$, then $\ell_{\phi}(w)$ is not an $\mathscr{L}_{1}$-space.

PROOF. As we have already noticed $\ell_{\phi}(w)$ is isometrically isomorphic to $\ell_{\psi}$, where $\Psi=\left(\psi_{n}\right)$ is a normalized Musielak-Orlicz function with $\psi_{n}(u)=\phi\left(a_{n} u\right) w_{n}$ and $a_{n}=\phi^{-1}\left(1 / w_{n}\right)$ for all $u \geq 0$ and $n \in \mathbb{N}$. By Theorem 4.1, we have $\ell_{1} \hookrightarrow \ell_{\Psi} \hookrightarrow \ell_{\infty}$. The condition $\phi \in \Delta_{2}$ implies that $\ell_{\phi}(w)$ is separable for any weight $w$. Thus if $\ell_{\phi}(w)$ were an $\mathscr{L}_{1}$-space, then $\ell_{\psi}^{*}$ would be isomorphic to $\ell_{\infty}$, by [22, Corollary 2]. Since $\ell_{\psi}^{*}=\ell_{\psi^{*}}$, a Banach lattice $E=\ell_{\psi^{*}}$ is isomorphic to AM-space $\ell_{\infty}$. Thus, by applying [1, Theorem 5], we would get that there exists a constant $C>0$ such that $\left\|x_{1}+\cdots+x_{n}\right\|_{E} \leq C \max \left\{\left\|x_{1}\right\|_{E}, \ldots,\left\|x_{n}\right\|_{E}\right\}$ for any pairwise disjoint elements $x_{1}, \ldots, x_{n} \in E$. Since $\ell_{1} \hookrightarrow \ell_{\Psi^{*}} \hookrightarrow \ell_{\infty}$, we have $\sup _{n}\left\|e_{n}\right\|_{E}<\infty$. In consequence, by the Fatou property of $E$, we conclude that the sequence with all terms equal to one belongs to $E=\ell_{\Psi^{*}}$. Since $\Psi^{*}=\left(w_{n} \phi^{*}\left(u / a_{n} w_{n}\right)\right)$,

$$
\sum_{n} w_{n} \phi^{*}\left(\frac{\lambda}{a_{n} w_{n}}\right)<\infty
$$


for some $\lambda>0$, which is impossible because $a_{n_{k}} w_{n_{k}} \rightarrow 0$ and $\sum_{k} w_{n_{k}}=\infty$ for some $\left(n_{k}\right) \subset \mathbb{N}$.

Note also that the above example and the result below show that there are MusielakOrlicz sequence spaces $\ell_{\Phi}$ with the Schur property, however $\Phi^{*}$ does not satisfy the condition ( $\mathrm{S})$.

PROPOSITION 5.6. Let $\Phi=\left(\phi_{n}\right)$ be such that $\phi_{n}(u)=\phi\left(a_{n} u\right) w_{n}$ with $a_{n}=$ $\phi^{-1}\left(1 / w_{n}\right)$ and $\left(w_{n}\right) \in \Lambda$. Then $\Phi^{*}$ satisfies condition (S) if and only if $\phi(u) \asymp u$ on $\mathbb{R}_{+}$.

PROOF. Without loss of generality we assume that $\lim _{n} w_{n}=0$ and $\sum_{n=1}^{\infty} w_{n}=\infty$. Assume that (S) is satisfied for $\Phi^{*}$. If $\Phi^{*}$ is a finite valued function, $\lim _{u \rightarrow \infty} \phi(u) / u=$ $\infty$. Let $\delta>0,0<\lambda<1,\left(A_{j}\right) \in \ell_{1}^{+}$and $\left(n_{j}\right) \subset \mathbb{N}$ be arbitrary. By Lemma 4.6, $\inf _{n} b_{n}=c>0$, where $\phi_{n}^{*}\left(b_{n}\right)=\delta$ and $\phi_{n}^{*}(u)=w_{n} \phi^{*}\left(u / a_{n} w_{n}\right)$. Hence

$$
\begin{aligned}
C_{i j} & =\sup _{\phi_{i}^{*}(u) \leq \delta}\left\{\phi^{*}\left(\lambda \frac{u}{a_{i} w_{i}}\right)-A_{j} \phi^{*}\left(\frac{u}{a_{i} w_{i}}\right)\right\} \\
& \geq \sup _{0 \leq u \leq c}\left\{\phi^{*}\left(\lambda \frac{u}{a_{i} w_{i}}\right)-A_{j} \phi^{*}\left(\frac{u}{a_{i} w_{i}}\right)\right\} \\
& =\sup _{0 \leq u \leq c / a_{i} w_{i}}\left\{\phi^{*}(\lambda u)-A_{j} \phi^{*}(u)\right\} .
\end{aligned}
$$

We have $\lim _{i} a_{i} w_{i}=\lim _{i} \phi^{-1}\left(1 / w_{i}\right) /\left(1 / w_{i}\right)=0$. Let $[0, M]$ be such a large interval that $\phi^{*}(\lambda u)>0$ for some $u \in[0, M]$. There exists $k \in \mathbb{N}$ such that $c / a_{i} w_{i}>M$ for $i \geq k$. Then $C_{i j} \geq C_{j}$ for all $i \geq k$ and $j \in \mathbb{N}$, where $C_{j}=\sup _{0 \leq u \leq M}\left\{\phi^{*}(\lambda u)-\right.$ $\left.A_{j} \phi^{*}(u)\right\}$. It is clear that $C_{j}>0$ for sufficiently large $j$. Hence

$$
\sum_{j=1}^{\infty} \sum_{i \geq n_{j}} w_{i} C_{i j} \geq \sum_{j=1}^{\infty} \sum_{i \geq k \vee n_{j}} w_{i} C_{j}=\sum_{j=1}^{\infty} C_{j} \sum_{i \geq k \vee n_{j}} w_{i}=\infty .
$$

This contradicts condition (S). Hence $\phi^{*}$ admits some infinite values, which yields that $A=\lim _{u \rightarrow \infty} \phi(u) / u<\infty$. Again let (S) be satisfied and assume that $\phi^{*}(u)>0$ for all $u>0$. Let further $a=\sup \left\{u: \phi^{*}(u)<\infty\right\}$ and $t_{n}=\sup \left\{u: \phi_{n}^{*}(u) \leq \delta\right\}$. Since $0<a<\infty$, for $i$ large enough $a_{i} w_{i} a / 2 \leq t_{i} \leq a a_{i} w_{i}$. In view of $\lim _{i} a_{i} w_{i}=1 / A$, there exist $k \in \mathbb{N}$ and $d>0$ such that $t_{i} \geq d$ for all $i \geq k$. Hence, analogously as before, for $i \geq k$

$$
C_{i j} \geq \sup _{0 \leq u \leq d / a_{i} w_{i}}\left\{\phi^{*}(\lambda u)-A_{j} \phi^{*}(u)\right\}
$$

Now choose $m>k$ such that for $i \geq m, c / a_{i} w_{i} \geq A C / 2$. It follows that for $i \geq m$,

$$
C_{i j} \geq \sup _{0 \leq u \leq A C / 2}\left\{\phi^{*}(\lambda u)-A_{j} \phi^{*}(u)\right\},
$$


which again contradicts $(S)$, since the right-hand term of the above inequality is positive in view of the assumption that $\phi^{*}(u)>0$ for every $u>0$. Thus $\lim _{u \rightarrow 0} \phi(u) / u>0$, and combining with $\lim _{u \rightarrow \infty} \phi(u) / u<\infty$, we obtain that $\phi(u) \asymp u$ on $\mathbb{R}_{+}$. Now if $\phi(u)=u$, then $\phi^{*}(u)=0$ for $0 \leq u \leq 1$ and $\phi^{*}(u)=\infty$ for $u>1$. Hence $\phi^{*}$ satisfies (S) trivially. Since $(S)$ is preserved under equivalence, the proof is complete.

We finish this section with the result which shows that the Schur property of a weighted Orlicz sequence space does not imply that its symmetrization is isomorphic to $\ell_{1}$, and that the assumption in Corollary 3.6 that $\left(e_{n}\right)$ is a semi-normalized basis is essential.

Recall that if $\phi$ is a finite valued Orlicz function which vanishes only at zero and $w=$ $\left(w_{n}\right) \in \Lambda$ is a non-increasing sequence, then the Orlicz-Lorentz symmetric sequence space $\lambda_{\phi, w}$ consists of all real sequences $x=(x(n))$ such that $\sum_{n=1}^{\infty} \phi\left(\epsilon x^{*}(n)\right) w_{n}<\infty$ for some $\epsilon>0$ and is equipped with the norm

$$
\|x\|=\inf \left\{\epsilon>0: \sum_{n=1}^{\infty} \phi\left(x^{*}(n) / \epsilon\right) w_{n} \leq 1\right\} .
$$

If $\phi(t)=t^{p}, 1 \leq p<\infty, \lambda_{\phi, w}$ coincides with the classical Lorentz space $d(w, p)$ (see [23]).

COROLlARY 5.7. Let $w=\left(w_{n}\right) \in \Lambda$ and let $\phi$ be a finite valued Orlicz function which vanishes only at zero. Then the following statements hold true:

(i) $\left(\lambda_{\phi, v}\right)_{a} \hookrightarrow S\left(\ell_{\phi}(w)_{a}\right)$ with $v=\left(v_{n}\right)$, where $v_{n}=\sup _{k \geq n}\left|w_{k}\right|$;

(ii) if $\left(w_{n}\right)$ is non-increasing and $\ell_{\phi}(w)$ has the Schur property, then $S\left(\dot{\ell}_{\phi}(w)\right)=$ $d(w, 1)$ is not isomorphic to $\ell_{1}$ and the continuous inclusion map $\left(\ell_{1}, d(w, 1)\right)_{\theta, p} \hookrightarrow$ $\ell_{\phi}(w)$ is compact for any $0<\theta<1$ and $1<p<\infty$.

PROOF. (i) Obviously $v=\left(v_{n}\right)$ is non-increasing, $v \in \Lambda$, and $\ell_{\phi}(v) \hookrightarrow \ell_{\phi}(w)$. Then the required result follows by the following easily verified equality

$$
\sup _{\pi \in \Pi} \sum_{n=1}^{\infty} \phi\left(\left|x_{\pi(n)}\right|\right) v_{n}=\sum_{n=1}^{\infty} \phi\left(x^{*}(n)\right) v_{n},
$$

which holds for any $x \in \lambda_{\phi, v}$.

(ii) Since $\ell_{\phi}(w) \in(\mathrm{SP})$, we have by Theorem 5.3, $\phi \in \Delta_{2}$ and $\phi(t) \asymp t$ on $[0,1]$. This yields by (i) that $S\left(\ell_{\phi}(w)\right)=\lambda_{\phi, w}=d(w, 1)$ up to equivalence of norms. Since $e_{n} \rightarrow 0$ weakly in $d(w, 1)$, we conclude that $d(w, 1) \notin(\mathrm{SP})$, and so $S\left(\ell_{\phi}(w)\right)$ is not isomorphic to $\ell_{1}$. Moreover the continuous inclusion map $\ell_{1} \hookrightarrow d(w, 1)$ is weakly compact (see proof of Proposition 3.4). This implies by [5] that $\left(\ell_{1}, d(w, 1)\right)_{\theta, p}$ is reflexive space for any $0<\theta<1$ and $1<p<\infty$. Since $\ell_{\phi}(w) \in(\mathrm{SP})$ and $\left(\ell_{1}, d(w, 1)\right)_{\theta, p} \hookrightarrow \ell_{\phi}(w)$, the result follows. 


\section{Weak Dunford-Pettis and positive Schur properties in Orlicz spaces}

We conclude the paper with a characterization of the weak Dunford-Pettis and positive Schur properties in Orlicz spaces defined on any non-atomic infinite measure space. As far as we know there is no characterization of the weak Dunford-Pettis property in Orlicz spaces defined on a finite measure space. It is easy to show (see proof below) that if the measure space $(\Omega, \mathscr{B}, \mu)$ is non-atomic and finite (respectively, infinite), then $L_{\phi}(\mu) \in$ (wDP) if and only if $\ell_{\phi}(w) \in$ (wDP) for any $w \in \Lambda_{1}$ (respectively, for some or equivalently for any $w \in \Lambda$ ). In particular, it follows from Theorem 5.1 that $L_{\phi}(\mu) \in$ (wDP) with $\phi(u)=u \ln (1+u)$ for any finite measure space (see [20]).

In the case of infinite non-atomic measure spaces we have the following result.

THEOREM 6.1. Let $\phi$ be a finite valued Orlicz function which vanishes only at zero and let $L_{\phi}(\mu)$ be an Orlicz space over a non-atomic and infinite measure space $(\Omega, \mathscr{B}, \mu)$. Then the following statements are equivalent:

(i) $L_{\phi}(\mu)$ has the positive Schur property;

(ii) $L_{\phi}(\mu)$ has the weak Dunford-Pettis property;

(iii) $\phi \in \Delta_{2}, \phi(t) \asymp t$ on $[0,1]$ and $\psi(t) \asymp t$ on $[0,1]$ for every $\psi \in C_{\phi}^{\infty}$;

(iv) $\phi \in \Delta_{2}$ and $\psi(t) \asymp t$ on $[0,1]$ for every $\psi \in C_{\phi}(0, \infty)$.

ProOF. Implication from (i) to (ii) is obvious and the equivalence of (iii) and (iv) follows from Theorem 5.3.

(ii) implies (iii). It is clear that complemented sublattices of a Banach lattice with the (wDP)- property, have this property too. Take any sequence $\left(\Omega_{n}\right)$ of mutually disjoint measurable sets in $\mathscr{B}$ such that $w=\left(w_{n}\right) \in \Lambda$ with $w_{n}=\mu\left(\Omega_{n}\right)$ and observe that the map $P$ defined by

$$
P f=\sum_{n=1}^{\infty} \frac{1}{\mu\left(\Omega_{n}\right)}\left(\int_{\Omega_{n}} f d \mu\right) \chi \Omega_{n}
$$

is the contractive projection which maps $L_{\phi}(\mu)$ onto $\left[\chi_{\Omega_{n}}\right]_{n=1}^{\infty}$. It is clear that the map $\left(x_{n}\right) \mapsto \sum_{n} x_{n} \chi_{\Omega_{n}}$ is an order isometry of $\ell_{\phi}(w)$ onto a sublattice $\left[\chi_{\Omega_{n}}\right]_{n=1}^{\infty}$ of $L_{\phi}(\mu)$. This implies that $\ell_{\phi}(w)$ has the weak Dunford-Pettis property, and thus also the Schur property [34]. In consequence, (ii) holds by Theorem 5.3.

(iii) implies (i). By [34] it is enough to show that every disjoint, weakly null sequence in $L_{\phi}(\mu)$ converges in norm. Thus, take a disjoint weakly null sequence $\left(f_{n}\right)$ in $L_{\phi}(\mu)$. Since $\phi \in \Delta_{2}$, the set of simple functions is dense in $L_{\phi}(\mu)$. Let $g_{n}=\sum_{k \in I_{n}} x_{n}^{k} \chi_{A_{n}^{k}}$, where $\left(I_{n}\right)$ is a sequence of finite subsets of $\mathbb{N}$ and $\left(A_{n}^{i}\right)_{i \in I_{n}, n \in \mathbb{N}}$ is a sequence of mutually disjoint measurable sets of $\mathscr{B}$ of positive measure such 
that $\sum_{n=1}^{\infty}\left\|f_{n}-g_{n}\right\|_{L_{\phi}(\mu)}<1 / 2$. From [23, Proposition 1.a.9], it follows that the basic sequences $\left(f_{n}\right)$ and $\left(g_{n}\right)$ are equivalent. Following [29, proof of Theorem 2.7], define the weight sequence $v=\left(v_{n, i}\right)=\left(\mu\left(A_{n}^{i}\right)\right)_{i \in I_{n}, n \in \mathbb{N}}$. Clearly, $\ell_{\phi}(v)$ is order isometrically isomorphic to $\left[g_{n}\right]_{n=1}^{\infty}$ in $L_{\phi}(\mu)$. By the Darboux property of the series $\sum_{n} w_{n}$, we conclude that $\ell_{\phi}(v)$ is order isometrically isomorphic to a sublattice of $\ell_{\phi}(w)$. Now by Theorem 5.3, we have $\ell_{\phi}(w) \in(\mathrm{SP})$. In consequence, $\left[g_{n}\right]_{n=1}^{\infty} \in(\mathrm{SP})$. From the above inequality we get that $g_{n} \rightarrow 0$ weakly in $L_{\phi}(\mu)$, by $f_{n} \rightarrow 0$ weakly. Hence $g_{n} \rightarrow 0$ in $L_{\phi}(\mu)$ yields $f_{n} \rightarrow 0$ (by the above inequality), and the proof is complete.

REMARK. Notice that the assumptions in the above theorem that $\phi$ is finite-valued and vanishes only at zero are essential. In fact it is easy to check that up to equivalence of norms, $L_{\phi}(\mu)=L_{1}(\mu) \cap L_{\infty}(\mu)$ whenever $\phi(t)=0$ for $0 \leq t \leq 1$ and $\phi(t)=\infty$ for $t>1$, and $L_{\phi}(\mu)=L_{1}(\mu)+L_{\infty}(\mu)$ whenever $\phi(t)=0$ for $0 \leq t \leq 1$ and $\phi(t)=t-1$ for $t>1$. In [15] it is shown that these spaces defined on any nonatomic separable measure space have (DP)-property and hence also (wDP)-property. However, it is clear that these spaces fail to have the positive Schur property.

\section{Acknowledgements}

We thank Nigel Kalton for valuable comments during preparation of the paper.

\section{References}

[1] Y. A. Abramovich and P. Wojtaszczyk, 'The uniqueness of the order in the spaces $L^{P}[0,1]$ and $\ell^{p}$, Mat. Zametki 18 (1975), 313-325 (in Russian).

[2] C. D. Aliprantis and O. Burkinshaw, Positive operators (Academic Press, New York, 1985).

[3] C. R. Banerjee and B. K. Lahiri, 'On subseries of divergent series', Amer. Math. Monthly 71 (1964), $767-768$.

[4] J. Bergh and J. Löfström, Interpolation spaces. An introduction, Grundhlehren der Math. Wissen. 223 (Springer, Berlin, 1976).

[5] W. J. Davis, T. Figiel, W. B. Johnson and A. Pełczyński, 'Factoring weakly compact operators', J. Funct. Anal. 17 (1974), 311-327.

[6] J. Diestel, 'A survey of results related to the Dunford-Pettis property', in: Proceedings of the Conference on Integration. Topology, and Geometry in Linear Spaces (Univ. North Carolina, Chapel Hill, N.C., 1979) (Amer. Math. Soc., Providence, R.I., 1980) pp. 15-60.

[7] L. Drewnowski, ' $F$-spaces with a basis which is shrinking but not hyper-shrinking', Studia Math. 64 (1979), 97-104.

[8] I. Halperin and H. Nakano, 'Generalized $\ell_{p}$-spaces and the Schur property', J. Math. Soc. Japan 5 (1953), 50-58.

[9] F. L. Hernández and V. Peirats, 'Weighted sequence subspaces of Orlicz function spaces isomorphic to $\ell_{p}$, Arch. Math. 50 (1988), 270-280. 
[10] F. L. Hernández and C. Ruiz, ‘On Musielak-Orlicz spaces isomorphic to Orlicz spaces', Comment. Math. Prace Mat. 32 (1992), 55-60.

[11] H. Hudzik and A. Kamińska, 'On uniformly convexifiable and B-convex Musielak-Orlicz spaces', Comment. Math. Prace Mat. 25 (1985), 59-75.

[12] S. Janson, P. Nilsson and J. Peetre, 'Notes on Wolff's note on interpolation spaces', Proc. London Math. Soc. 48 (1984), 283-299.

[13] W. B. Johnson, B. Maurey, G. Schechtman and L. Tzafriri, 'Symmetric structures in Banach spaces', Mem. Amer. Math. Soc. (217) 19 (1979).

[14] A. Kamińska, 'Indices, convexity and concavity in Musielak-Orlicz spaces', Funct. Approx. Comment. Math. 26 (1998), 67-84.

[15] A. Kamińska and M. Mastyło, 'The Dunford-Pettis property for symmetric spaces', Canad. $J$. Math. 52 (2000), 789-803.

[16] L. V. Kantorovich and G. P. Akilov, Functional analysis, 2nd revised edition, (Nauka, Moscow, 1977). English translation: (Pergamon Press, Oxford, 1982).

[17] E. Katirtzoglou, 'Type and cotype in Musielak-Orlicz spaces', J. Math. Anal. Appl. 226 (1998), $431-455$.

[18] M. A. Krasnoselskii and Ya. B. Rutickii, Convex functions and Orlicz spaces (Nordhoff, Groningen, 1961).

[19] S. G. Kreĭn, Ju. I. Petunin and E. M. Semenov, Interpolation of linear operators (Nauka, Moscow, 1978). English translation: (Amer. Math. Soc., Providence, R.I., 1982).

[20] D. Leung, 'On the weak Dunford-Pettis property', Arch. Math. 52 (1989), 363-364.

[21] M. Levy, 'L'espace d'interpolation réel $\left(A_{0}, A_{1}\right)_{\theta, p}$ contient $\ell_{p}$ ', C. R. Acad. Sci. Paris 289 (1979), 675-677.

[22] J. Lindenstrauss and A. Pełczyński, 'Absolutely summing operators in $\mathscr{L}_{p}$-spaces and their applications', Studia Math. 29 (1968), 275-326.

[23] J. Lindenstrauss and L. Tzafriri, Classical Banach spaces, Vol. I, II (Springer, Berlin, 1977, 1979).

[24] L. Maligranda, 'Indices and interpolation', Dissertationes Math. (Rozprawy Mat.) 234 (1985), 49.

[25] M. Mastyło, 'The universal right $K$ property for some interpolation spaces', Studia Math. 90 (1988), 117-128.

[26] J. Musielak, Orlicz spaces and modular spaces (Springer, Berlin, 1983).

[27] N. J. Nielsen, 'On the Orlicz function spaces $L^{M}(0, \infty)$ ', Israel J. Math. 20 (1975), 237-259.

[28] S. Ya. Novikov, 'Singularities of embedding operators between symmetric function spaces on [0, 1]', Math. Notes 62 (1997), 457-468.

[29] V. Peirats and C. Ruiz, 'On $\ell^{p}$-copies in Musielak-Orlicz sequence spaces', Arch. Math. 58 (1992), $164-173$.

[30] F. Räbiger, 'Lower and upper 2-estimates for order bounded sequences and Dunford-Pettis operators between certain classes of Banach lattices', in: Functional analysis (Austin, TX, 1987/1989), Lect. Notes in Math. 1470 (Springer, Berlin, 1991) pp. 159-170.

[31] W. L. C. Sargent, 'Some sequence spaces related to the $\ell^{p}$ spaces', J. London Math. Soc. 35 (1960), 161-171.

[32] W. Wnuk, ' $l p_{n}^{\left(p_{n}\right)}$ spaces with the Dunford-Pettis property', Comment. Math. Prace Mat. 30 (1991), 483-489.

[33] —- 'Banach lattices with properties of the Schur type-a survey', Confer. Sem. Mat. Univ. Bari No. 249 (1993), 25 pages.

[34] _ , 'Banach lattices with the weak Dunford-Pettis property', Atti Sem. Mat. Fis. Univ. Modena 42 (1994), 227-236.

[35] J. Woo, 'On modular sequence spaces', Studia Math. 58 (1973), 271-289.

[36] S. Yamamuro, 'Modulared sequence spaces', J. Fac. Sci. Hokkaido Univ. Ser. I. 13 (1954), 1-12. 
Department of Mathematical Sciences

The University of Memphis

Memphis TN 38152

USA

e-mail: kaminska@memphis.edu
Faculty of Mathematics and

Computer Science

A. Mickiewicz University

and

Institute of Mathematics

Poznań Branch

Polish Academy of Sciences

Matejki 48/49

60-769 Poznań

Poland

e-mail: mastylo@amu.edu.pl 